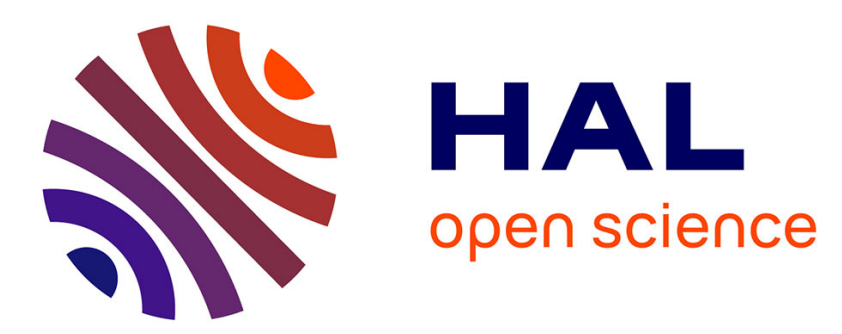

\title{
Exponential stability of a network of serially connected Euler-Bernoulli beams
}

Denis Mercier, Virginie Régnier

\section{To cite this version:}

Denis Mercier, Virginie Régnier. Exponential stability of a network of serially connected Euler-Bernoulli beams. International Journal of Control, 2014, 87 (6), pp.1266-1281. 10.1080/00207179.2013.874597 . hal-03163679

\section{HAL Id: hal-03163679 \\ https://uphf.hal.science/hal-03163679}

Submitted on 28 Sep 2021

HAL is a multi-disciplinary open access archive for the deposit and dissemination of scientific research documents, whether they are published or not. The documents may come from teaching and research institutions in France or abroad, or from public or private research centers.
L'archive ouverte pluridisciplinaire HAL, est destinée au dépôt et à la diffusion de documents scientifiques de niveau recherche, publiés ou non, émanant des établissements d'enseignement et de recherche français ou étrangers, des laboratoires publics ou privés. 


\title{
Exponential stability of a network of serially connected Euler-Bernoulli beams
}

\author{
D. Mercier, V. Régnier *
}

December 9, 2013

\begin{abstract}
The aim is to prove the exponential stability of a system modelling the vibrations of a network of $N$ Euler-Bernoulli beams serially connected. Using a result due to $\mathrm{K}$. Ammari and M. Tucsnak, the problem is reduced to the estimate of a transfer function and the obtention of an observability inequality. The solution is then expressed in terms of Fourier series so that one of the sufficient conditions for both the estimate of the transfer function and the observability inequality is that the distance between two consecutive large eigenvalues of the spatial operator involved in this evolution problem is superior to a minimal fixed value. This property called spectral gap holds. It is proved using the exterior matrix method due to W. H. Paulsen. Two more asymptotic estimates involving the eigenfunctions are required. They are established using an adequate basis.
\end{abstract}

Key words Network, Beams, Stability, Spectral gap, Exterior matrices.

AMS 34B45, 74K10, 93B60, 93D15.

\section{Introduction}

In the last few years various physical models of multi-link flexible structures consisting of finitely many interconnected flexible elements such as strings, beams, plates, shells have been mathematically studied. See the references by Ali Mehmeti, von Below and Nicaise in [16] as well as [10] and [13], for instance. The spectral analysis of such structures has some applications to control or stabilization problems (cf. [13]).

For interconnected strings (corresponding to a second-order operator on each string), a lot of results have been obtained: the asymptotic behaviour of the eigenvalues (see the references by Ali Mehmeti, von Below and Nicaise in [16]), the relationship between the eigenvalues and

*Laboratoire de Mathématiques et ses Applications de Valenciennes, FR CNRS 2956, Institut des Sciences et Techniques de Valenciennes, Université de Valenciennes et du Hainaut-Cambrésis, Le Mont Houy, 59313 VALENCIENNES Cedex 9, FRANCE, e-mails : denis.mercier@univ-valenciennes.fr ; virginie.regnier@univvalenciennes.fr 
algebraic theory (cf. papers by von Below, Nicaise and [13]), qualitative properties of solutions (see papers by von Below cited in [16] for example) etc...

For interconnected beams (corresponding to a fourth-order operator on each beam), some results on the asymptotic behaviour of the eigenvalues and on the relationship between the eigenvalues and algebraic theory were obtained by Nicaise and Dekoninck with different kinds of connections using the method developed by von Below in [8] to get the characteristic equation associated to the eigenvalues.

The authors used the same method in [15] to compute the spectrum for a hybrid system of $N$ flexible beams connected by $n$ vibrating point masses. This type of structure was studied by Castro and Zuazua in many papers (see [9] and the papers by the same authors cited in [16] as well one by Castro and Hansen also cited there).

In another paper (see [16]), the authors used the technique of exterior matrices due to W. H. Paulsen (presented for other purposes in [18]) which D. Mercier had already used in the same type of context in [14]. The aim of these papers was to establish controllability. The technique of exterior matrices used in [16] helped us prove controllability in a more general context than what we had done in [15]. This is why we keep this method for the present paper.

In a joint work by the authors and K. Ammari and J. Valein, the stabilization of a chain of Euler-Bernoulli beams and strings was proved using a spectral analysis also based on the exterior matrices technique (see [6]).

In this paper we will investigate the same problem as in [10]. In that paper, Chen and al. have established the exponential stability of the problem but with an assumption on the material constants (the mass densities are supposed to be decreasing and the flexural rigidities must be increasing). They remark that the assumption makes the beam more flexible at the extremity with the control and seem to think that the exponential stability could not hold without this assumption.

Our purpose is to prove, using another method (that of the exterior matrices - they have used a moment method), that in fact, the exponential stability always holds. The problem is different from the one we had studied in [16]. A feedback law is added but the interior masses have disappeared. Moreover we prove the exponential stability of the problem and not only its controllability.

The network we consider is a chain of $N$ serially connected branches $(N \geq 2)$ with $n=N+1$ vertices (denoted by $E_{i}$ ).

Let us call $\left(P_{K}\right)$ the stability problem:

$$
\begin{array}{rlrl}
m_{j} u_{j, t t}(x, t)+a_{j} u_{j}^{(4)}(x, t) & =0, & & \forall j \in\{1, \ldots, N\}, \\
u_{1}(0, t)=u_{1}^{(1)}(0, t) & =0, & & \\
u_{j}\left(l_{j}, t\right)-u_{j+1}(0, t) & =0, & & \forall j \in\{1, \ldots, N-1\}, \\
u_{j}^{(1)}\left(l_{j}, t\right)-u_{j+1}^{(1)}(0, t) & =0, & \forall j \in\{1, \ldots, N-1\},
\end{array}
$$




$$
\begin{aligned}
a_{j} u_{j}^{(2)}\left(l_{j}, t\right)-a_{j+1} u_{j+1}^{(2)}(0, t)=0, & \forall j \in\{1, \ldots, N-1\}, \\
a_{j} u_{j}^{(3)}\left(l_{j}, t\right)-a_{j+1} u_{j+1}^{(3)}(0, t)=0, & \forall j \in\{1, \ldots, N-1\}, \\
a_{N} u_{N}^{(2)}\left(l_{N}, t\right)=0, & \\
a_{N} u_{N}^{(3)}\left(l_{N}, t\right)=z(t) . &
\end{aligned}
$$

where the feedback law is chosen as :

$$
z(t)=K u_{N, t}\left(l_{N}, t\right)
$$

where $K>0, t \geq 0$.

The scalar function $u_{j}(x, t)$ contains the information on the vertical displacement of the $j$ th beam. This displacement is described by the first equation where $m_{j}$ is the constant mass density of the $j$-th beam and $a_{j}$ its flexural rigidity $(1 \leq j \leq N)$.

The third, fourth, fifth and sixth equations are transmission conditions. The second, seventh and eighth ones are boundary conditions. Note that the damping function $z=z(t)$ acts on the system through the exterior node $E_{N}$ on the quantity $a_{N} u_{N}^{(3)}\left(l_{N}, t\right)$.

The goal of the paper is to establish on the first hand the existence and uniqueness of the solution of Problem $\left(P_{K}\right)$ with a regularity depending on that of the initial conditions, on the other hand, that the energy of the solution decays to zero exponentially.

Before starting to study the core of the problem, we apply in Section 2 the terminology of networks to our particular network. The whole terminology can be found in early contributions of Lumer and Gramsch as well as in papers by Ali Mehmeti ([1] and [2]), von Below (cf. [8]) and Nicaise ([17] and [3]) in the eighties. We also give some properties of the spatial operator $A$ involved in the considered evolution problem and construct an operator $B$ and its adjoint to rewrite the problem as the abstract evolution equation (28). The aim is to apply the results of the paper by Ammari and Tucsnak ([4] and also [5]).

In Section 3, we give the main results: two properties of the eigenelements of the operator $A$. One called the spectral gap concerns the asymptotic behaviour of the difference between two consecutive eigenvalues, the other one is an estimate from both above and below of the value of an eigenfunction at $x=l_{N}$.

Both results are sufficient conditions to establish the estimate of the transfer function required by Ammari and Tucsnak's method (cf. [4] and [5]) as well as the observability inequality called (2.5) in their paper and rewritten in our context as (44). The exponential stability of the problem follows.

The proof of the spectral gap (asymptotic behaviour of the difference between two consecutive eigenvalues) is given in Section 4. This asymptotic behaviour is given by that of the roots of a function called $f_{\infty}$. In order to avoid the cancellation of the large order terms, the characteristic equation is computed using the exterior matrix method due to Paulsen (see [18]) and already used by D. Mercier in [14]. 
Two estimates involving the evaluation of an eigenfunction of the problem at the node where the damping acts as well as a norm of the same eigenfunction remain to be proved. It is the aim of Section 5. The choice of the basis $h_{i}$ (cf. the notation at the beginning of Section 5) in which the eigenfunctions are decomposed is crucial for the asymptotic behaviour of the eigenfunctions to be studied since the expressions are very complicated especially for large values of $N$. In particular the exponential factor in $h_{3}$ has an important role since its presence keeps the exponential terms from being disseminated in the different matrices which would not allow an easy estimation of the involved quantities as $\lambda$ tends to infinity.

\section{Data and Framework}

\subsection{Domain and notation}

The domain that we consider is a network of $N(N \in \mathbb{N}-\{0,1\})$ serially connected beams which can be modelled by a graph $G=\bigcup_{j=1}^{N} k_{j}$. Each branch $k_{j}$ having an origin and an end such that the end of the branch $k_{j}(1 \leq j \leq N-1)$ is connected to the beginning of the branch $k_{j+1}$. By the intermediary of a parametrization we will identify each branch $k_{j}$ with the interval $\left[0, l_{j}\right], 0$ represents the beginning of $k_{j}$ and $l_{j}$ the end. For each branch $k_{j}$, we fix mechanical constants $m_{j}>0$ (the mass density of the beam $k_{j}$ ) and $a_{j}>0$ (the flexural rigidity of $k_{j}$ ). The vibration of the branch $k_{j}$ is modelled by the function $u_{j}(t, x), t \geq 0, x \in\left[0, l_{j}\right], j=1, \ldots, N$. The total vibration of the structure is the vectorial function $u=\left(u_{j}\right)_{j=1, \ldots, N}$.

Notation for derivatives. In this paper, for a function $u=u(x, t)$ we make the choice to denote by $u_{t}\left(u_{t t}, \ldots,\right)$ the first $($ second,$\ldots)$ time derivative and $u^{(1)}\left(u^{(2)}, \ldots\right)$ the first (second,...) spatial derivative.

\subsection{The stability problem}

We assume that each beam is uniform, with constant mass density $m_{j}$ and flexural rigidity $a_{j}, j=1, \ldots, N$. The problem, denoted by $\left(P_{K}\right)$, is the following:

$$
\begin{aligned}
m_{j} u_{j, t t}(x, t)+a_{j} u_{j}^{(4)}(x, t) & =0, & & \forall j \in\{1, \ldots, N\}, \\
u_{1}(0, t)=u_{1}^{(1)}(0, t) & =0, & & \\
u_{j}\left(l_{j}, t\right)-u_{j+1}(0, t) & =0, & & \forall j \in\{1, \ldots, N-1\}, \\
u_{j}^{(1)}\left(l_{j}, t\right)-u_{j+1}^{(1)}(0, t) & =0, & & \forall j \in\{1, \ldots, N-1\}, \\
a_{j} u_{j}^{(2)}\left(l_{j}, t\right)-a_{j+1} u_{j+1}^{(2)}(0, t) & =0, & & \forall j \in\{1, \ldots, N-1\}, \\
a_{j} u_{j}^{(3)}\left(l_{j}, t\right)-a_{j+1} u_{j+1}^{(3)}(0, t) & =0, & & \forall j \in\{1, \ldots, N-1\},
\end{aligned}
$$




$$
\begin{array}{r}
a_{N} u_{N}^{(2)}\left(l_{N}, t\right)=0, \\
a_{N} u_{N}^{(3)}\left(l_{N}, t\right)=z(t) .
\end{array}
$$

The feedback law is chosen as :

$$
z(t)=K u_{N, t}\left(l_{N}, t\right)
$$

where $K>0, t \geq 0$.

\subsection{Abstract framework}

In order to study the above system $\left(P_{K}\right)$ we need to formulate it in an abstract setting. More precisely, we shall see that the framework given in [4] is well adapted to our problem $\left(P_{K}\right)$.

\subsubsection{The operator $A$ : definition and properties}

For that purpose, we define the Hilbert space

$$
\mathcal{H}=\prod_{j=1}^{N} L^{2}\left(0, l_{j}\right)
$$

endowed by the inner product

$$
(u, \tilde{u})_{\mathcal{H}}=\sum_{j=1}^{N} m_{j} \int_{0}^{l_{j}}<u_{j}(x), \tilde{u}_{j}(x)>d x,
$$

where $<., .>$ represents the Hermitian product in $\mathbb{C}$.

We also define the space $V$

$$
V=\left\{u \in \prod_{j=1}^{N} H^{2}\left(0, l_{j}\right) \text { satisfying (19) to }(21) \text { hereafter }\right\},
$$

$$
\begin{aligned}
u_{1}(0)=u_{1}^{(1)}(0)=0, & \\
u_{j}\left(l_{j}\right)-u_{j+1}(0)=0, & \forall j \in\{1, \ldots, N-1\}, \\
u_{j}^{(1)}\left(l_{j}\right)-u_{j+1}^{(1)}(0)=0, & \forall j \in\{1, \ldots, N-1\},
\end{aligned}
$$

as well as the sesquilinear form $a(u, v)$ for $(u, v) \in V \times V$ by

$$
a(u, v)=\sum_{j=1}^{N} a_{j} \int_{0}^{l_{j}}<u_{j}^{(2)}(x), v_{j}^{(2)}(x)>d x .
$$

Next, we define the linear operator $A: D(A) \longrightarrow \mathcal{H}$ by

$$
A u=\left(\frac{a_{j}}{m_{j}} u_{j}^{(4)}\right)_{1 \leq j \leq N}
$$


with domain

$$
D(A)=\left\{u \in \mathcal{H}: u \in\left(\prod_{j=1}^{N} H^{4}\left(0, l_{j}\right)\right) \cap V, \text { satisfying (23) to (26) hereafter }\right\}
$$

$$
\begin{array}{rlrl}
a_{j} u_{j}^{(2)}\left(l_{j}\right)-a_{j+1} u_{j+1}^{(2)}(0) & =0, & \forall j \in\{1, \ldots, N-1\}, \\
a_{j} u_{j}^{(3)}\left(l_{j}\right)-a_{j+1} u_{j+1}^{(3)}(0) & =0, \quad \forall j \in\{1, \ldots, N-1\}, \\
a_{N} u_{N}^{(2)}\left(l_{N}\right) & =0, & \\
a_{N} u_{N}^{(3)}\left(l_{N}\right) & =0 . &
\end{array}
$$

The operator $A$ is a linear unbounded self-adjoint and strictly positive operator in $\mathcal{H}$. The domain of $A^{\frac{1}{2}}$ is $D\left(A^{\frac{1}{2}}\right)=V$ (the proof based on Friedrichs extension is left to the reader).

\subsubsection{The operators $B$ and $B^{*}$}

We define the operator $B: \mathbb{C} \longrightarrow V^{\prime}$ such that $\forall z \in \mathbb{C}, B z=A C z$, where $V^{\prime}$ is the dual space of $V$ with respect to the inner product of the pivot space $\mathcal{H}$ and $C z=u$ is the solution of

$$
u_{i}^{(4)}=0, i=1, \ldots, N
$$

which satisfies conditions $(19),(20),(21),(23),(24),(25)$ as well as the following condition

$$
a_{N} u_{N}^{(3)}\left(l_{N}\right)=-z .
$$

Then its adjoint $B^{*}: V \longrightarrow \mathbb{C}$ is defined by $B^{*} \Phi=\Phi_{N}\left(l_{N}\right)$, for any $\Phi \in V$ and the system $\left(P_{K}\right)$ is described by

$$
u_{t t}(t)+A u(t)+K \cdot B B^{*} u_{t}(t), u(0)=u^{0}, u_{t}(0)=u^{1}, t \in[0, \infty) .
$$

with $u(t) \in V$. A solution of (28) is $u$ such that $u(t) \in V$ for $t \in[0, \infty)$ and, for any $\Phi \in V$ :

$$
\begin{aligned}
& \left(u_{t t}(t), \Phi\right)_{\mathcal{H}}+a(u(t), \Phi)+K a\left(C B^{*}\left(u_{t}(t)\right), \Phi\right)=0 \Longleftrightarrow \sum_{j=1}^{N} m_{j} \int_{0}^{l_{j}}<u_{j, t t}(t, x), \Phi_{j}(x)>d x \\
& +\sum_{j=1}^{N} a_{j} \int_{0}^{l_{j}}<u_{j}^{(2)}(t, x), \Phi_{j}^{(2)}(x)>d x+K \sum_{j=1}^{N} a_{j} \int_{0}^{l_{j}}<p_{j}^{(2)}(t, x), \Phi_{j}^{(2)}(x)>d x=0
\end{aligned}
$$

where $p=\left(p_{1}, \ldots, p_{N}\right)$ is the solution of

$$
p_{i}^{(4)}=0, i=1, \ldots, N
$$

which satisfies conditions (19), (20), (21), (23), (24), (25) and $a_{N} p_{N}^{(3)}\left(l_{N}\right)=-u_{N, t}\left(l_{N}, t\right)$.

Two integrations by parts and adapted choices for $\Phi$ lead to the equivalence between Problem $\left(P_{K}\right)$ and the abstract rewriting (28). Thus, the framework given in [4] is well adapted to our problem $\left(P_{K}\right)$ (see equations $(1.3),(1.4)$ in [4]). 


\subsubsection{The dissipative operator $A_{d}$}

The dissipative operator $A_{d}$ introduced by Ammari and Tucsnak ([4]) in the same paper is still $\mathcal{A}_{d}: D\left(\mathcal{A}_{d}\right) \longrightarrow V \times \mathcal{H}$ defined by

$$
\mathcal{A}_{d}=\left(\begin{array}{cc}
0 & I \\
-A & -K \cdot B B^{*}
\end{array}\right)
$$

with domain

$$
D\left(\mathcal{A}_{d}\right)=\left\{(u, v) \in V \times \mathcal{H}: A u+K \cdot B B^{*} v \in \mathcal{H}, v \in V\right\} .
$$

Thus the abstract equation (28) can be rewritten as

$$
w_{t}(t)=\mathcal{A}_{d} w(t), w(0)=w^{0}, t \in[0, \infty)
$$

with $w(t) \in D\left(\mathcal{A}_{d}\right), w^{0}=\left(u^{0}, u^{1}\right)$.

Let us state some classical results based on Lumer-Phillips Theorem.

Proposition 2.1 (existence and uniqueness of the solution, decreasing of the energy)

1. Assume that $w^{0}=\left(u^{0}, u^{1}\right) \in D\left(\mathcal{A}_{d}\right)$. Then equation (30) has a unique solution

$$
w \in C\left(0, \infty, D\left(\mathcal{A}_{d}\right)\right) \cap C^{1}(0, \infty ; V \times \mathcal{H}) .
$$

Thus equation (28) has a unique solution

$$
u \in C^{1}(0, \infty ; V)
$$

such that $B^{*} u(\cdot) \in H^{1}(0, T ; \mathbb{C})$ and

$$
\left\|B^{*} u_{t}\right\|_{L^{2}(0, T ; \mathbb{C})}^{2} \leq C\left\|\left(u^{0}, u^{1}\right)\right\|_{V \times \mathcal{H}}^{2}
$$

where the constant $C>0$ is independent of $\left(u^{0}, u^{1}\right)$.

2. We still assume that $w^{0}=\left(u^{0}, u^{1}\right) \in D\left(\mathcal{A}_{d}\right)$. The energy of the solution $u(t)$ given above, defined by

$$
E(u(t))=\frac{1}{2}\left\{\left\|u_{t}(t)\right\|_{\mathcal{H}}^{2}+\left\|A^{1 / 2} u(t)\right\|_{\mathcal{H}}^{2}\right\}
$$

satisfies

$$
E(u(0))-E(u(t))=\int_{0}^{t}\left|B^{*} u_{t}(s)\right|^{2} d s \geq 0, \forall t \geq 0
$$




\section{Main results}

\subsection{Properties of the eigenelements of the operator $A$}

Consider the eigenvalue problem: $\lambda^{2} \in \sigma(A)(\lambda>0)$ is an eigenvalue of $A$ with associated eigenvector $\phi \in D(A)$ if and only if $\phi$ satisfies the transmission and boundary conditions (19), (20), (21), (23), (24), (25), (26) of Section 2.3.1 and

$$
(E P) \begin{cases}\phi_{j}^{(4)}=q_{j}^{4} \lambda^{2} \phi_{j} & \text { on }\left(0,1_{j}\right), \forall \mathrm{j} \in\{1, \ldots, \mathrm{N}\}, \\ \phi_{j} \in H^{4}\left(\left(0, l_{j}\right)\right), & \forall j \in\{1, \ldots, N\},\end{cases}
$$

with $q_{j}=\left(m_{j} / a_{j}\right)^{1 / 4}$.

The following results are useful for stability. They are proved in Sections 4 and 5 respectively.

Theorem 3.1 (the spectral gap)

Let $\lambda_{k}^{2}, k \in \mathbb{N}^{*},\left(\lambda_{k}>0\right)$ be the (strictly) monotone increasing sequence of eigenvalues of Problem $(E P)$ given above then

$$
\lim _{k \rightarrow+\infty}\left(\lambda_{k+1}-\lambda_{k}\right)=+\infty
$$

Theorem 3.2 (uniform estimates for $\left|\phi_{N}\left(l_{N}\right)\right|$ )

Consider the eigenvalue problem $(E P)$ given above. For any eigenfunction $\phi \in D(A)$ associated to the eigenvalue $\lambda^{2}$ and for any $K>0$, there exists two constants $K_{1}$ and $K_{2}$ such that:

$$
K_{1} \cdot\|\phi\|_{\mathcal{H}}^{2} \leq\left|\phi_{N}\left(l_{N}\right)\right|^{2} \leq K_{2} \cdot\|\phi\|_{\mathcal{H}}^{2}
$$

with the norm $\|\cdot\|_{\mathcal{H}}$ introduced in Section 2.3.1.

\subsection{Strong stability}

Using the abstract framework given in last section, we prove the decay to zero of the energy of any solution of the abstract equation (30) with suitable initial condition. It is enough to establish that the operator $\mathcal{A}_{d}$ (introduced in Definition 2.3.3) has no eigenvalues on the imaginary axis.

Theorem 3.3 (strong stability)

It holds

$$
\lim _{t \rightarrow+\infty} E(u(t))=0
$$

for any solution $w=(u, v)$ of the abstract equation (30) with $w^{0}$ in $V \times \mathcal{H}$.

Proof. First we compute $\Re\left[\left(\mathcal{A}_{d} w, w\right)_{V \times \mathcal{H}}\right]$. 
$\begin{aligned}\left.{ }_{(39}\right)^{\left(\mathcal{A}_{d} w, w\right)_{V \times \mathcal{H}}} & =(v, u)_{V}+\left(-A u-K \cdot B B^{*} v, v\right)_{\mathcal{H}}=a(v, u)-(A u, v)_{\mathcal{H}}-K\left(B B^{*} v, v\right)_{\mathcal{H}} \\ & =\overline{a(u, v)}-a(u, v)-K\left|B^{*} v\right|^{2}=-2 i \Im(a(u, v))-K\left|v_{N}\left(l_{N}\right)\right|^{2} .\end{aligned}$

Thus $\Re\left[\left(\mathcal{A}_{d} w, w\right)_{V \times \mathcal{H}}\right]=-K\left|v_{N}\left(l_{N}\right)\right|^{2}$.

Now we want to prove that the operator $\mathcal{A}_{d}$ has no eigenvalues of the form $i \mu$, with $\mu \in \mathbb{R}$. To this end, we suppose that there exists $w=(u, v) \neq(0,0)$ in $D\left(\mathcal{A}_{d}\right)$, such that $\mathcal{A}_{d} w=i \mu w$. Then, by definition of $\mathcal{A}_{d}$, it holds:

$$
\left\{\begin{array}{l}
v=i \mu u \\
A u+K \cdot B B^{*} v=-i \mu v
\end{array}\right.
$$

Now for such a $w, \Re\left[\left(\mathcal{A}_{d} w, w\right)_{V \times \mathcal{H}}\right]=\Re\left[i \mu(w, w)_{V \times \mathcal{H}}\right]=0$. It follows $v_{N}\left(l_{N}\right)=0$. Since $v_{N}\left(l_{N}\right)=B^{*} v$, system $(40)$ implies $u_{N}\left(l_{N}\right)=0$ and $A u=\mu^{2} u$. Moreover, since $(u, v) \in D\left(\mathcal{A}_{d}\right)$, $A u+K \cdot B B^{*} v \in \mathcal{H}$ i.e. $A u \in \mathcal{H}\left(\right.$ since $\left.B^{*} v=v_{N}\left(l_{N}\right)=0\right)$. Thus $u \in D(A)$.

Two cases must be envisaged:

- either $\mu \neq 0$. In that case, any eigenfunction of the conservative operator $A$ satisfies $u_{N}\left(l_{N}\right) \neq 0$ (see Theorem 3.2). This is a contradiction with our assumption.

- Or $\mu=0$. Then, if $u$ is an eigenfunction associated to the eigenvalue $\mu^{2}=0$,

$$
0=(A u, u)_{\mathcal{H}}=a(u, u)=\sum_{j=1}^{N} a_{j} \int_{0}^{l_{j}}<u_{j}^{(2)}(x), u_{j}^{(2)}(x)>d x
$$

which implies $u_{j}^{(2)}=0$ on $\left(0 ; l_{j}\right)$ for any $j$ in $\{0, \ldots, N\}$. From condition (11), it follows $u_{1}=0$ (indeed $u_{1}$ must be a polynomial function with degree 1 which vanishes as well as its first derivative at 0$)$. Then conditions (12) and (13) combined with $u_{1}\left(l_{1}\right)=u_{2}(0)=0$ and $u_{2}^{(2)}=0$ on $\left(0 ; l_{2}\right)$ imply $u_{2}=0$ and so on: $u_{j}=0$ on $\left(0 ; l_{j}\right)$ for any $j$ in $\{0, \ldots, N\}$. This is a contradiction.

The conclusion is that $\mathcal{A}_{d}$ has no eigenvalues of the form $i \mu$, with $\mu \in \mathbb{R}$. The result follows, using the main theorem of [7].

\subsection{Exponential stability}

Using Theorem 2.2 of [4] we can state the exponential stability of our system. To this end, we prove (1.5) of [4] (also called hypothesis $(H)$, which is the estimate of a transfer function) directly (using the orthonormal basis formed by the eigenfunctions of the operator $A$ ) as well as the estimate (2.5) which is an observability inequality. To establish $(H)$, we need the spectral gap and since the proof of the spectral gap is a long and technical proof based on the same ideas as those we have already used in a previous paper (cf. [16]), it is given in next section. As for the observability inequality (2.5), its proof requires both the spectral gap and a uniform estimate for $\left|\phi_{N}\left(l_{N}\right)\right|$ which is proved via an adaptation of ancient results (same paper [16]). It is also given in another section. Note that some new technical difficulties appear in the 
calculations and proofs of Sections 4 and 5 and that, although they are based on the same ideas as a previous paper, they are not trivial at all.

Theorem 3.4 (exponential stability)

The system described by the abstract equation (30) is exponentially stable in $V \times \mathcal{H}$.

Proof. To prove this theorem, we use Theorem 2.2 of [4]. In the first part of the proof, we will check that the following hypothesis $(H)$ is satisfied:

(H): If $\beta>0$ is fixed and $C_{\beta}=\{\lambda \in \mathbb{C} \mid \Re(\lambda)=\beta\}$, the function

$$
\lambda \in C_{\beta} \rightarrow H(\lambda)=\lambda B^{*}\left(\lambda^{2}+A\right)^{-1} B \in \mathcal{L}(\mathbb{C})
$$

is bounded.

Since the proof is long, we divide it into several steps.

First step: rewrite $H(\lambda)$ as a series. We start by computing $B(1)$ ( $B$ is a linear operator defined on $\mathbb{C}$ thus computing $B(1)$ is enough to know any value $B(\lambda)$ ).

Since $B(1) \in V^{\prime}$ then there exists a sequence $\left(\alpha_{k}\right)_{k \in \mathbb{N}^{*}}$ such that

$$
\sum_{k=1}^{\infty}\left|\frac{\alpha_{k}}{\lambda_{k}}\right|^{2}<\infty \text { and } B(1)=\sum_{k=1}^{\infty} \alpha_{k} \phi_{k} .
$$

(with $V$ introduced in Definition 2.3.1, $\left(\phi_{k}\right)_{k}$ the orthonormal basis formed by the eigenvectors of the operator $A$ and $\lambda_{k}^{2}$ the eigenvalues).

Let $h=\sum_{k=1}^{\infty} h_{k} \phi_{k}$ any element of $V$ (i.e $\left.\sum_{k=1}^{\infty}\left|h_{k} \lambda_{k}\right|^{2}<\infty\right)$ then

$$
<B(1), h>_{V^{\prime}, V}=\sum_{k=1}^{\infty}<\alpha_{k}, h_{k}>\text {. }
$$

We also have

$$
<B(1), h>_{V^{\prime}, V}=<1, B^{*} h>_{\mathbb{C}, \mathbb{C}}=\overline{h_{N}\left(l_{N}\right)}=\sum_{k=1}^{\infty} \overline{h_{k}} \overline{\phi_{k, N}\left(l_{N}\right)} .
$$

From (41) and (42) we deduce that

$$
B(1)=\sum_{k=1}^{\infty} \overline{\phi_{k, N}\left(l_{N}\right)} \phi_{k}
$$

Now, as previously, we directly compute $\left(\lambda^{2} I+A\right)^{-1} B(1)$ in the orthonormal basis $\left(\phi_{k}\right)_{k}$ and easily find that

$$
\left(\lambda^{2} I+A\right)^{-1} B(1)=\sum_{k=1}^{\infty} \frac{\overline{\phi_{k, N}\left(l_{N}\right)}}{\lambda^{2}+\lambda_{k}^{2}} \phi_{k} .
$$

Therefore

$$
H(\lambda)=\lambda B^{*}\left(\lambda^{2} I+A\right)^{-1} B(1)=\lambda \sum_{k=1}^{\infty} \frac{\left|\phi_{k, N}\left(l_{N}\right)\right|^{2}}{\lambda^{2}+\lambda_{k}^{2}} .
$$


Second step: find an estimate for the imaginary part of $H(\lambda) / \lambda$ on the line $C_{\beta}$. We can choose $\beta=1$ without loss of generality. Calculating the imaginary part of $H(1+i y) /(1+i y)$ leads to:

$$
-\frac{1}{2 y} \Im\left(\frac{H(\lambda)}{\lambda}\right)=\sum_{k=1}^{\infty}\left|\phi_{k, N}\left(l_{N}\right)\right|^{2} \cdot f_{1}\left(y, \lambda_{k}\right), \text { for } \lambda=1+i y, y \in \mathbb{R} .
$$

where $f_{1}(y, \lambda)=\frac{1}{4 y^{2}+\left(1-y^{2}+\lambda^{2}\right)^{2}}$.

Due to Theorem 3.2, $K_{1} \leq\left|\phi_{k, N}\left(l_{N}\right)\right|^{2} \leq K_{2}$ (the norm of each $\phi_{k}$ in $\mathcal{H}$ is one).

Thus, the function we need to estimate from above is the function of $y$ defined by

$$
\Sigma(y)=\sum_{k=1}^{\infty} \frac{1}{4 y^{2}+\left(1-y^{2}+\lambda^{2}\right)^{2}} .
$$

For a fixed $y>0$, the sum $\Sigma$ is separated into three terms: one with the small values of $\lambda_{k}$, one with the values of $\lambda_{k}$ which are close to the value of $y$ (these values contribute mostly to the sum but their number is finite) and the last one with the big values of $\lambda_{k}$.

1. By definition, $\Sigma_{1}(y)=\sum_{k: \lambda_{k} \leq y-\sqrt{y}} \frac{1}{4 y^{2}+\left(1-y^{2}+\lambda^{2}\right)^{2}}$. The function $f_{1}$ is an increasing function of $\lambda$ as soon as $y$ is large enough. Indeed the derivative $\partial_{\lambda} f_{1}(y, \lambda)$ is equal to

$$
\frac{4 \lambda\left(y^{2}-\lambda^{2}-1\right)}{\left(4 y^{2}+\left(1-y^{2}+\lambda^{2}\right)^{2}\right)^{2}} \text {. }
$$

Then, since $\lambda \leq y-\sqrt{y}, f_{1}(y, \lambda) \leq f_{1}(y, y-\sqrt{y})$ and

$$
f_{1}(y, y-\sqrt{y})=\frac{1}{4 y^{2}+\left(1-y^{2}+(y-\sqrt{y})^{2}\right)^{2}} \lesssim \frac{1}{y^{4}}
$$

Note that the notation $A \lesssim B$ means the existence of a positive constant $C$, which is independent of $A$ and $B$ such that $A \leq C B$.

Now, from the spectral gap (cf. Theorem 3.1), $\lambda_{k} \gtrsim k^{2}$. Then, denoting by $\mathcal{N}_{y}$, the number of $\lambda_{k}$ such that $\lambda_{k} \leq y-\sqrt{y}$, it holds:

$$
\left(\mathcal{N}_{y}\right)^{2} \lesssim \lambda_{\mathcal{N}_{y}} \leq y-\sqrt{y}
$$

Thus, for large values of $y, \Sigma_{1}(y) \lesssim \mathcal{N}_{y} \frac{1}{y^{4}} \lesssim \frac{\sqrt{y}}{y^{4}} \lesssim \frac{1}{y^{2}}$.

2. By definition, $\Sigma_{2}(y)=\sum_{k: y-\sqrt{y} \leq \lambda_{k} y+\sqrt{y}} \frac{1}{4 y^{2}+\left(1-y^{2}+\lambda^{2}\right)^{2}}$.

First $f_{1}(y, \lambda) \leq \frac{1}{4 y^{2}}$ 
Secondly, the number $N_{y}$ which is the number of $\lambda_{k}$ such that $y-\sqrt{y} \leq \lambda_{k} \leq y+\sqrt{y}$ is bounded as regards $y$. Indeed $N_{y}$ is smaller than the quotient of the amplitude of the interval $(2 \sqrt{y})$ over the minimum gap between two consecutive values of $\lambda_{k}$ (with $y-\sqrt{y} \leq \lambda_{k} \leq y+\sqrt{y}$ ). Now the gap between two values of $\lambda_{k}$ is:

$$
\begin{aligned}
\lambda_{k+1}-\lambda_{k} & =\left(\sqrt{\lambda_{k+1}}-\sqrt{\lambda_{k}}\right)\left(\sqrt{\lambda_{k+1}}+\sqrt{\lambda_{k}}\right) \\
& \geq \sigma\left(\sqrt{\lambda_{k+1}}+\sqrt{\lambda_{k}}\right) \geq 2 \sigma \sqrt{\lambda_{k}} \gtrsim k
\end{aligned}
$$

where the parameter $\sigma$ is introduced in the proof of Theorem 4.10.

Now, we define $k_{0}$ the largest integer such that $\lambda_{k_{0}} \leq y-\sqrt{y}$. Then, for any $k$ such that $y-\sqrt{y} \leq \lambda_{k} \leq y+\sqrt{y}, \lambda_{k+1}-\lambda_{k} \gtrsim k_{0}$. Thus $N_{y} \lesssim \frac{2 \sqrt{y}}{k_{0}}$.

Moreover there exists $\gamma_{2}>0, \lambda_{k} \leq \gamma_{2} k^{2}$ (cf. [11]) and since $y-\sqrt{y} \leq \lambda_{k_{0}+1} \leq \gamma_{2}\left(k_{0}+1\right)^{2}$, $k_{0} \geq \sqrt{\frac{y-\sqrt{y}}{\gamma_{2}}}-1$ and $N_{y} \lesssim \frac{2 \sqrt{y}}{\sqrt{y}} \lesssim 1$.

The conclusion is: $\Sigma_{2}(y) \lesssim \frac{1}{y^{2}}$.

3. By definition, $\Sigma_{3}(y)=\sum_{k: y+\sqrt{y} \leq \lambda_{k}} \frac{1}{4 y^{2}+\left(1-y^{2}+\lambda^{2}\right)^{2}}$. The derivative $\partial_{y} f_{1}(y, \lambda)$ is equal to

$$
\frac{4 y\left(\lambda^{2}-y^{2}-1\right)}{\left(4 y^{2}+\left(1-y^{2}+\lambda^{2}\right)^{2}\right)^{2}}
$$

If $\lambda \geq y+\sqrt{y}$, it is positive then $f_{1}(y, \lambda) \leq f_{1}(y(\lambda), \lambda)$ with $y(\lambda)+\sqrt{y(\lambda)}=\lambda$ and $y(\lambda) \leq \lambda$ i.e. $y(\lambda)=\frac{1}{2}(2 \lambda+1-\sqrt{4 \lambda+1})$. Since $\lambda_{k} \gtrsim k^{2}$ (as it was said above), the series $\sum_{k} \frac{1}{\lambda_{k}}$ is convergent and it follows, after calculations:

$$
f_{1}(y, \lambda) \lesssim \frac{1}{\lambda^{3}} \text { which implies } \Sigma_{3}(y) \lesssim \frac{1}{y^{2}} \sum_{k: y+\sqrt{y} \leq \lambda_{k}} \frac{1}{\lambda_{k}} \lesssim \frac{1}{y^{2}} .
$$

The conclusion of this part is that the imaginary part of $H(\lambda) / \lambda$ satisfies:

$$
\left|\Im\left(\frac{H(\lambda)}{\lambda}\right)\right| \lesssim \frac{1}{y}, \text { for any } \lambda \in C_{\beta}=\{\lambda \in \mathbb{C} \mid \Re(\lambda)=\beta\}
$$

Third step: find an estimate for the real part of $H(\lambda) / \lambda$ on the line $C_{\beta}$. We still choose $\beta=1$ and calculate the real part of $H(1+i y) /(1+i y)$. It is:

$$
\Re\left(\frac{H(\lambda)}{\lambda}\right)=\sum_{k=1}^{\infty}\left|\phi_{k, N}\left(l_{N}\right)\right|^{2} \cdot f_{2}\left(y, \lambda_{k}\right), \text { for } \lambda=1+i y, y \in \mathbb{R} .
$$


where $f_{2}(y, \lambda)=\frac{1-y^{2}+\lambda^{2}}{4 y^{2}+\left(1-y^{2}+\lambda^{2}\right)^{2}}$. We still separate the sum into three terms denoted by $\Sigma_{1}^{\prime}, \Sigma_{2}^{\prime}$ and $\Sigma_{3}^{\prime}$.

Since the ideas of the proof are similar to those of the proof for the imaginary part, we give less details for this step.

For the estimate of $\Sigma_{1}^{\prime}$, we first prove that the derivative $\partial_{\lambda} f_{2}(y, \lambda)$ is negative if $\lambda \leq y-\sqrt{y}$ and $y$ is large enough. Thus $\left|f_{2}(y, \lambda)\right|=-f_{2}(y, \lambda) \leq-f_{2}(y, y-\sqrt{y}) \lesssim \frac{1}{y^{3 / 2}}$.

Now the number $\mathcal{N}_{y}$ of $\lambda_{k}$ such that $\lambda_{k} \leq y-\sqrt{y}$ satisfies $\mathcal{N}_{y} \lesssim \sqrt{y}$ since $\left(\mathcal{N}_{y}\right)^{2} \lesssim \lambda_{\mathcal{N}_{y}} \leq y-\sqrt{y}$. Thus $\Sigma_{1}(y) \lesssim \frac{1}{y}$.

For the estimate of $\Sigma_{2}^{\prime}$, we prove that the derivative $\partial_{\lambda} f_{2}(y, \lambda)$ vanishes at $\lambda$ such that $1+\lambda^{2}-y^{2}= \pm 2 y$ i.e. at $\lambda_{ \pm}=\sqrt{y^{2} \pm 2 y-1}$. Moreover, $f_{2}(y, y \pm \sqrt{y})=\frac{ \pm 1}{2 y \sqrt{y}}+o\left(\frac{ \pm 1}{2 y \sqrt{y}}\right)$. Since $f_{2}\left(y, \lambda_{ \pm}\right)=\frac{ \pm 1}{4 y}$, it holds, for any $\lambda$ in $[y-\sqrt{y} ; y+\sqrt{y}],\left|f_{2}(y, \lambda)\right| \leq\left|f_{2}\left(y, \lambda_{+}\right)\right| \lesssim \frac{1}{y}$. Thus $\Sigma_{2}^{\prime} \lesssim \frac{1}{y}$ (recall that $N_{y}$ is bounded, cf. the second step).

At last, for the estimate of $\Sigma_{3}^{\prime}$, we prove that the derivative $\partial_{y} f_{2}(y, \lambda)$ is positive if $\lambda \geq y+\sqrt{y}$ and $y$ is large enough. Thus, $f_{2}(y, \lambda) \leq f_{2}(\lambda, \lambda)$ and since $f_{2}(\lambda, \lambda)=\frac{1}{4 \lambda^{2}+1} \lesssim \frac{1}{\lambda^{2}}$, it follows:

$$
\Sigma_{3}^{\prime}(y) \lesssim \frac{1}{y} \sum_{k: y+\sqrt{y} \leq \lambda_{k}} \frac{1}{\lambda_{k}} \lesssim \frac{1}{y} .
$$

The conclusion of this part is that the real part of $H(\lambda) / \lambda$ satisfies:

$$
\left|\Re\left(\frac{H(\lambda)}{\lambda}\right)\right| \lesssim \frac{1}{y} \text {, for any } \lambda \in C_{\beta}=\{\lambda \in \mathbb{C} \mid \Re(\lambda)=\beta\} .
$$

The second assumption for the exponential stability is the observability inequality called (2.5) in the paper by Ammari and Tucsnak. It follows from the spectral gap and the uniform estimate given in Section 3.1 using a result due to Haraux (cf. [12]). Indeed (2.5) is:

$$
\exists T>0, \exists C(T), \int_{0}^{T}\left|v_{N}\left(l_{N}, t\right)\right|^{2} d t \geq C(T) \cdot\left\|U_{0}\right\|_{V \times H}^{2}
$$

where $U(t)=(u(t), v(t))^{t}$ satisfies $U^{\prime}=\mathcal{A}_{c} U, U(0)=U_{0} \in D\left(\mathcal{A}_{c}\right)$, with $\mathcal{A}_{c}$ the conservative operator defined like $\mathcal{A}_{d}$ with $K=0$.

Define $\Phi_{k}=\left(\phi_{k}, i \lambda_{k} \phi_{k}\right)^{t}$ an orthonormal basis of eigenfunctions of the operator $\mathcal{A}_{c}$ (in particular $\left\|\Phi_{k}\right\|_{V \times H}=1$ ). The result due to Haraux (cf. [12]) that we already used with more details in [16] allows to write: 


$$
\exists T>0, \exists C_{1}(T), \int_{0}^{T}\left|v_{N}\left(l_{N}, t\right)\right|^{2} d t \geq C_{1}(T) \sum_{k=1}^{\infty}\left|u_{0}^{k}\right|^{2}\left|\lambda_{k}\right|^{2}\left|\phi_{k, N}\left(l_{N}\right)\right|^{2}
$$

where $U_{0}=\sum_{k=1}^{\infty} u_{0}^{k} \Phi_{k}$. It follows from Theorem 3.2, $\left|\phi_{k, N}\left(l_{N}\right)\right|^{2} \geq K_{1}\left\|\phi_{k}\right\|_{H}^{2}$ and since there exists $C_{2}$ such that $\left\|\phi_{k}\right\|_{H}^{2} \geq C_{2} \frac{1}{\left|\lambda_{k}\right|^{2}}\left\|\Phi_{k}\right\|_{V \times H}^{2}$

$$
\exists T>0, \exists C_{1}(T), \exists C_{2}, \exists K_{1}, \int_{0}^{T}\left|v_{N}\left(l_{N}, t\right)\right|^{2} d t \geq C_{1}(T) C_{2} K_{1} \sum_{k=1}^{\infty}\left|u_{0}^{k}\right|^{2} .
$$

Assumption (44) follows with $C(T)=C_{1}(T) C_{2} K_{1}$ since $\left\|U_{0}\right\|_{V \times H}^{2}=\sum_{k=1}^{\infty}\left|u_{0}^{k}\right|^{2}$

\section{Proof of the spectral gap using exterior matrices}

The proof of the spectral gap follows the lines of the proof of our previous paper on the boundary controllability of a chain of serially connected Euler-Bernoulli beams with interior masses (cf. [16]). We also use the exterior matrix method due to W. H. Paulsen (see [18]).

We need to determine the asymptotic behaviour of the characteristic equation of the eigenvalue problem: $\lambda^{2} \in \sigma(A)(\lambda>0)$ is an eigenvalue of $A$ with associated eigenvector $\phi \in D(A)$ if and only if $\phi$ satisfies the transmission and boundary conditions (19), (20), (21), (23), (24), (25), (26) of Section 2.3.1 and

$$
(E P) \begin{cases}\phi_{j}^{(4)}=q_{j}^{4} \lambda^{2} \phi_{j} & \text { on }\left(0,1_{j}\right), \forall \mathrm{j} \in\{1, \ldots, \mathrm{N}\}, \\ \phi_{j} \in H^{4}\left(\left(0, l_{j}\right)\right), & \forall j \in\{1, \ldots, N\},\end{cases}
$$

with $q_{j}=\left(m_{j} / a_{j}\right)^{1 / 4}$.

\subsection{Recall of notation and of some properties}

Let $\phi$ be a non-trivial solution of the above eigenvalue problem $(E P)$ and $\lambda^{2}(\lambda>0)$ be the corresponding eigenvalue.

For each $j \in\{1, \ldots, N\}$, the vector function $V_{j}$ is defined by

$$
V_{j}(x)=\left(\phi_{j}(x), \frac{\phi_{j}^{(1)}(x)}{\sqrt{\lambda}}, a_{j} \frac{\phi_{j}^{(2)}(x)}{\lambda}, a_{j} \frac{\phi_{j}^{(3)}(x)}{\lambda \sqrt{\lambda}}\right)^{t}, \forall x \in\left[0, l_{j}\right] .
$$

Keeping the notation $a_{j}$ and $l_{j}$ introduced in Section 2 , the matrix $A_{j}$ is $A_{j}:=A\left(q_{j}, b_{j}, m_{j}\right)$ with $q_{j}=\left(m_{j} / a_{j}\right)^{1 / 4}, b_{j}=q_{j} l_{j}$ and $A(q, b, m)$ the square matrix of order 4 defined by 


$$
A(q, b, m)=\frac{1}{2}\left(\begin{array}{cccc}
c h+c & \frac{s h+s}{q} & \frac{q^{2}(c h-c)}{m} & \frac{q(s h-s)}{m} \\
q(s h-s) & c h+c & \frac{q^{3}(s h+s)}{m} & \frac{q^{2}(c h-c)}{m} \\
\frac{m(c h-c)}{q^{2}} & \frac{m(s h-s)}{q^{3}} & c h+c & \frac{s h+s}{q} \\
\frac{m(s h+s)}{q} & \frac{m(c h-c)}{q^{2}} & q(s h-s) & c h+c
\end{array}\right)
$$

with the notation $c=\cos (b \sqrt{\lambda}), s=\sin (b \sqrt{\lambda}), c h=\cosh (b \sqrt{\lambda}), s h=\sinh (b \sqrt{\lambda})$.

To finish with, the matrix $M(\lambda)$ is given by

$$
M(\lambda)=A_{N} A_{N-1} \ldots A_{2} A_{1} .
$$

Lemma 4.1 (a few trivial but useful properties)

With the notation introduced above:

$$
\begin{aligned}
& V_{j}\left(l_{j}\right)=A_{j} V_{j}(0), \forall j \in\{1, \ldots, N\}, \\
& V_{j+1}(0)=V_{j}\left(l_{j}\right), \forall j \in\{1, \ldots, N-1\}, \\
& V_{N}\left(l_{N}\right)=M(\lambda) V_{1}(0) .
\end{aligned}
$$

The proof is analogous to that of [16].

Theorem 4.2 (the characteristic equation for the eigenvalue problem corresponding to a chain of $N$ branches)

$\lambda^{2}>0$ is an eigenvalue of $A$ if and only if $\lambda$ satisfies the characteristic equation

$$
f(\sqrt{\lambda})=\operatorname{det}\left(M_{22}(\lambda)\right)=0
$$

where $M_{22}(\lambda)$ is the square matrix of order 2 which is the restriction of the matrix $M(\lambda)$, given by (48), to its last two lines and its last two columns.

For that property again, the proof is similar to that of [16].

\subsection{Rewriting of the characteristic equation using the exterior matrix method}

The exterior matrix method presented in [18] is a very useful method which allows to compute asymptotically the eigenfrequencies for the vibrations of serially connected elements which are governed by fourth-order equations. But our goal is to get the spectral gap. The main idea is to exploit the special properties of the exterior matrices associated to our problem in order to obtain the desired results.

The whole section makes use of the same ideas as in a previous paper by D. Mercier (see [14]).

First, we simply recall the definition of exterior matrix and some useful results that we need in the sequel (see [18] for more details). 
Definition 4.3 If $M=\left(m_{i j}\right)$ is a $4 \times 4$ matrix, then the exterior matrix of $M$ is the $6 \times 6$ matrix given by:

$$
\operatorname{ext}(M)=\left(\begin{array}{ll}
\operatorname{ext}(M)_{11} & \operatorname{ext}(M)_{12} \\
\operatorname{ext}(M)_{21} & \operatorname{ext}(M)_{22}
\end{array}\right)
$$

where each block $\operatorname{ext}(M)_{i j}, i, j=1,2$, is a $3 \times 3$ matrix given hereafter:

$$
\begin{aligned}
& \operatorname{ext}(M)_{11}=\left(\begin{array}{ll}
\left|\begin{array}{ll}
m_{11} & m_{12} \\
m_{21} & m_{22}
\end{array}\right|\left|\begin{array}{ll}
m_{13} & m_{12} \\
m_{21} & m_{23}
\end{array}\right|\left|\begin{array}{ll}
m_{11} & m_{14} \\
m_{21} & m_{24}
\end{array}\right| \\
\left.\left|\begin{array}{ll}
m_{11} & m_{12} \\
m_{31} & m_{32}
\end{array}\right|\left|\begin{array}{ll}
m_{11} & m_{13} \\
m_{31} & m_{33}
\end{array}\right|\left|\begin{array}{ll}
m_{11} & m_{14} \\
m_{31} & m_{34}
\end{array}\right|\right), \\
\left.\left|\begin{array}{ll}
m_{11} & m_{12} \\
m_{41} & m_{42}
\end{array}\right|\left|\begin{array}{ll}
m_{11} & m_{13} \\
m_{41} & m_{43}
\end{array}\right|\left|\begin{array}{ll}
m_{11} & m_{14} \\
m_{41} & m_{44}
\end{array}\right|\right)
\end{array}\right. \\
& \operatorname{ext}(M)_{12}=\left(\begin{array}{ll}
\left|\begin{array}{ll}
m_{13} & m_{14} \\
m_{23} & m_{24}
\end{array}\right|-\left|\begin{array}{ll}
m_{12} & m_{14} \\
m_{22} & m_{24}
\end{array}\right|\left|\begin{array}{ll}
m_{12} & m_{13} \\
m_{22} & m_{23}
\end{array}\right| \\
\left|\begin{array}{ll}
m_{13} & m_{14} \\
m_{33} & m_{34}
\end{array}\right|-\left|\begin{array}{ll}
m_{12} & m_{14} \\
m_{32} & m_{34}
\end{array}\right|\left|\begin{array}{ll}
m_{12} & m_{13} \\
m_{32} & m_{33}
\end{array}\right| \\
\left.\left|\begin{array}{ll}
m_{13} & m_{14} \\
m_{43} & m_{44}
\end{array}\right|-\left|\begin{array}{ll}
m_{12} & m_{14} \\
m_{42} & m_{44}
\end{array}\right|\left|\begin{array}{ll}
m_{12} & m_{13} \\
m_{42} & m_{43}
\end{array}\right|\right),
\end{array}\right.
\end{aligned}
$$

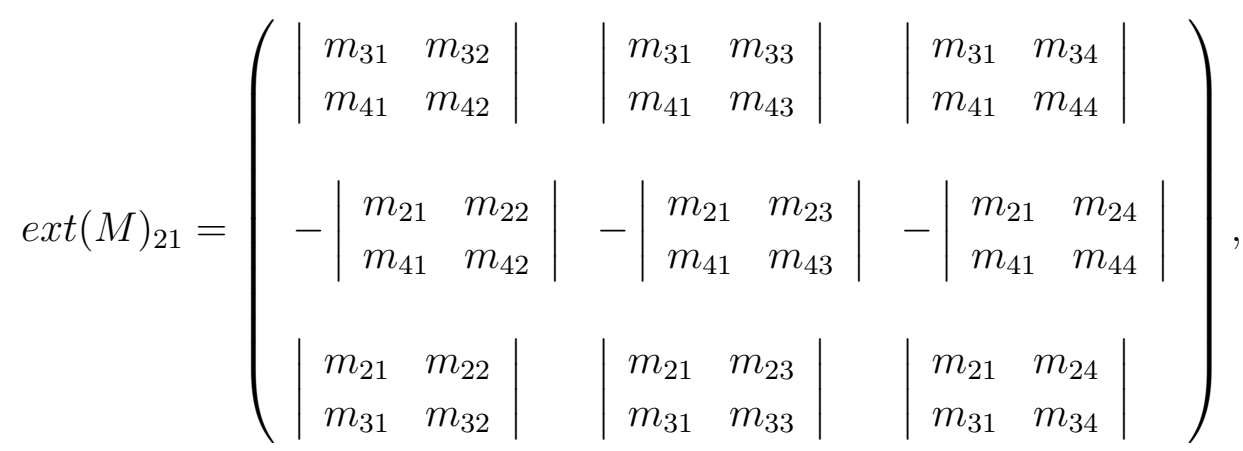




$$
\operatorname{ext}(M)_{22}=\left(\begin{array}{cc}
\left|\begin{array}{ll}
m_{33} & m_{34} \\
m_{43} & m_{44}
\end{array}\right| & -\left|\begin{array}{cc}
m_{32} & m_{34} \\
m_{42} & m_{44}
\end{array}\right|\left|\begin{array}{ll}
m_{32} & m_{33} \\
m_{42} & m_{43}
\end{array}\right| \\
-\left|\begin{array}{ll}
m_{23} & m_{24} \\
m_{43} & m_{44}
\end{array}\right|\left|\begin{array}{ll}
m_{22} & m_{24} \\
m_{42} & m_{44}
\end{array}\right| & -\left|\begin{array}{cc}
m_{22} & m_{23} \\
m_{42} & m_{43}
\end{array}\right| \\
\left|\begin{array}{ll}
m_{23} & m_{24} \\
m_{33} & m_{34}
\end{array}\right| & -\left|\begin{array}{cc}
m_{32} & m_{34} \\
m_{22} & m_{24}
\end{array}\right|\left|\begin{array}{ll}
m_{22} & m_{23} \\
m_{32} & m_{33}
\end{array}\right|
\end{array}\right) .
$$

Lemma 4.4 If $M_{1}$ and $M_{2}$ are $4 \times 4$ matrices, then

$$
\operatorname{ext}\left(M_{1} M_{2}\right)=\operatorname{ext}\left(M_{1}\right) \operatorname{ext}\left(M_{2}\right)
$$

Proof. Sketch of the proof (for more details see Lemma 1 of [18].)

Given a matrix $M \in \mathcal{M}_{4}(\mathbb{R})$, we define a linear map $M^{*}$ in $\mathcal{M}_{4}(\mathbb{R})$ such that :

$$
\forall A \in \mathcal{M}_{4}(\mathbb{R}), M^{*}(A)=M A M^{T} .
$$

It is easy to prove that the map $M \rightarrow M^{*}$ is a homomorphism (i.e we have $M_{1}^{*} M_{2}^{*}=\left(M_{1} M_{2}\right)^{*}$ ) and that $M^{*}$ sends anti-symmetric matrices to anti-symmetric matrices, so we can restrict $M^{*}$ to this subspace. A basis for the $4 \times 4$ anti-symmetric matrices is

$$
\begin{aligned}
& e_{1}=\left(\begin{array}{llll}
0 & 1 & 0 & 0 \\
-1 & 0 & 0 & 0 \\
0 & 0 & 0 & 0 \\
0 & 0 & 0 & 0
\end{array}\right), e_{2}=\left(\begin{array}{llll}
0 & 0 & 1 & 0 \\
0 & 0 & 0 & 0 \\
-1 & 0 & 0 & 0 \\
0 & 0 & 0 & 0
\end{array}\right), e_{3}=\left(\begin{array}{llll}
0 & 0 & 0 & 1 \\
0 & 0 & 0 & 0 \\
0 & 0 & 0 & 0 \\
-1 & 0 & 0 & 0
\end{array}\right), \\
& e_{4}=\left(\begin{array}{llll}
0 & 0 & 0 & 0 \\
0 & 0 & 0 & 0 \\
0 & 0 & 0 & 1 \\
0 & 0 & -1 & 0
\end{array}\right), e_{5}=\left(\begin{array}{llll}
0 & 0 & 0 & 0 \\
0 & 0 & 0 & -1 \\
0 & 0 & 0 & 0 \\
0 & 1 & 0 & 0
\end{array}\right), e_{6}=\left(\begin{array}{llll}
0 & 0 & 0 & 0 \\
0 & 0 & 1 & 0 \\
0 & -1 & 0 & 0 \\
0 & 0 & 0 & 0
\end{array}\right) .
\end{aligned}
$$

Using this basis, we find that $M^{*}$, when restricted to anti-symmetric matrices can be expressed by the $6 \times 6$ matrix $\operatorname{ext}(M)$ given in Definition 4.3 .

(50) expresses that the map $M \rightarrow M^{*}$ is a homomorphism.

Theorem 4.5 (the characteristic equation rewritten in terms of exterior matrices) Let $\lambda^{2}>0$ be an eigenvalue of $A$ then $\lambda$ satisfies the characteristic equation

$$
f(\sqrt{\lambda})=e_{4}^{t} \operatorname{ext}(M(\lambda)) e_{4}=0,
$$

or equivalently

$$
f(\sqrt{\lambda})=e_{4}^{t} \operatorname{ext}\left(A_{N}\right) \operatorname{ext}\left(A_{N-1}\right) \ldots \operatorname{ext}\left(A_{1}\right) e_{4}=0,
$$

where $M(\lambda)$ is the square matrix of order 4 given by (48) and $e_{4}^{t}=(0,0,0,1,0,0)$.

Proof. The proof is analogous to that of the paper [16]. The only difference is that the determinant of $M_{22}$ is the term of the 4-th line and 4-th column of the matrix $\operatorname{ext}(M)$. 


\subsection{The asymptotic behaviour of the characteristic equation}

As in [14], we study the asymptotic behaviour of the exterior matrices involved in $\operatorname{ext}(M(\lambda))$ in order to get the asymptotic behaviour of the characteristic equation (52) as $\lambda$ tends to $\infty$. This is enough to establish the following property called spectral gap. Let $\lambda_{k}^{2}, k \in \mathbb{N}^{*},\left(\lambda_{k}>0\right)$ be the (strictly) monotone increasing sequence of eigenvalues of Problem $(E P)$ given at the beginning of Section 4 then

$$
\lim _{k \rightarrow+\infty}\left(\lambda_{k+1}-\lambda_{k}\right)=+\infty
$$

Thus the aim of the following is to study the asymptotic behaviour of the exterior matrix of each matrix $A_{j}$ contained in the expression (48). From now on the notation $o(\lambda)$ is used for a square matrix of the appropriate size such that all its terms are dominated by the function $\lambda \mapsto \lambda$ asymptotically.

Definition 4.6 (definition of the matrices $C, S$ and of the vectors $V_{1}$ and $V_{2}$ )

$$
\begin{aligned}
& C(q, m)=\left(\begin{array}{cccccc}
1 & \frac{q^{3}}{m} & 0 & -\frac{q^{4}}{m^{2}} & \frac{q}{m} & 0 \\
\frac{m}{q^{3}} & 2 & \frac{1}{q} & -\frac{q}{m} & 0 & \frac{1}{q} \\
0 & q & 1 & 0 & -\frac{1}{q} & 1 \\
-\frac{m^{2}}{q^{4}} & -\frac{m}{q} & 0 & 1 & -\frac{m}{q^{3}} & 0 \\
\frac{m}{q} & 0 & -q & -\frac{q^{3}}{m} & 2 & -q \\
0 & q & 1 & 0 & -\frac{1}{q} & 1
\end{array}\right) . \\
& S(q, m)=\left(\begin{array}{cccccc}
0 & \frac{q^{3}}{m} & \frac{q^{2}}{m} & 0 & -\frac{q}{m} & \frac{q^{2}}{m} \\
-\frac{m}{q^{3}} & 0 & \frac{1}{q} & \frac{q}{m} & -\frac{2}{q^{2}} & \frac{1}{q} \\
-\frac{m}{q^{2}} & -q & 0 & \frac{q^{2}}{m} & -\frac{1}{q} & 0 \\
0 & -\frac{m}{q} & -\frac{m}{q^{2}} & 0 & \frac{m}{q^{3}} & -\frac{m}{q^{2}} \\
\frac{m}{q} & 2 q^{2} & q & -\frac{q^{3}}{m} & 0 & q \\
-\frac{m}{q^{2}} & -q & 0 & \frac{q^{2}}{m} & -\frac{1}{q} & 0
\end{array}\right) . \\
& V_{1}(q, m)=\left(-\frac{q^{4}}{m^{2}},-\frac{q}{m}, 0,1,-\frac{q^{3}}{m}, 0\right)^{t} . \\
& V_{2}(q, m)=\left(0, \frac{q}{m}, \frac{q^{2}}{m}, 0,-\frac{q^{3}}{m}, \frac{q^{2}}{m}\right)^{t} .
\end{aligned}
$$


Lemma 4.7 (properties of $H, C, S, V_{1}$ and $V_{2}$ )

The exterior matrix of $A_{j}$ may be written as:

$$
\operatorname{ext}\left(A_{j}\right)=e^{b_{j} \sqrt{\lambda}} H\left(q_{j}, b_{j}, m_{j}\right)+o\left(e^{b_{j} \sqrt{\lambda}}\right) .
$$

where the matrix $H$ is:

$$
\left.H\left(q_{j}, b_{j}, m_{j}\right)=\cos \left(b_{j} \sqrt{\lambda}\right) C\left(q_{j}, m_{j}\right)+\sin \left(b_{j} \sqrt{\lambda}\right) S\left(q_{j}, m_{j}\right)\right) .
$$

The rank of both matrices $C(q, m)$ and $S(q, m)$ defined above is 2 . A basis for the range of $C(q, m)$ is $\left\{V_{1}(q, m), V_{2}(q, m)\right\}$. Idem for the range of $S(q, m)$.

Proof. The first property is proved via a long calculation using the definition of exterior matrices. We do not give the details here. But for instance the first coefficient of $\operatorname{ext}\left(A_{j}\right)$ is :

$$
\frac{1}{2}\left(1+\cos \left(b_{j} \sqrt{\lambda}\right) \cdot \cosh \left(b_{j} \sqrt{\lambda}\right)\right)=\frac{e^{b_{j} \sqrt{\lambda}}}{4} \cos \left(b_{j} \sqrt{\lambda}\right)+o\left(e^{b_{j} \sqrt{\lambda}}\right) .
$$

A simple computation for the matrix $C(q, m)$ leads to $L_{3}=q \cdot\left(\frac{q^{3}}{m} L_{2}-L_{1}\right), L_{4}=-\frac{m^{2}}{q^{4}} L_{1}$, $L_{5}=\left(q^{2}+\frac{m}{q}\right) L_{1}-\frac{q^{5}}{m} L_{2}$ and $L_{6}=L_{3}$. And for the matrix $S(q, m), L_{2}=\frac{2 q^{3}}{m} L_{1}-\frac{1}{q^{2}} L_{5}$, $L_{3}=\frac{q^{4}}{m} L_{1}-\frac{1}{q} L_{5}, L_{4}=\frac{1}{q^{4}} L_{1}$ and $L_{6}=L_{3}$. Hence the rank is 2 for both matrices.

The vector $V_{1}$ is in the range of $C$ and $S$ since $V_{1}=C(0,0,0,1,0,0)^{t}$ and $V_{1}=S\left(0,0,0,1, q^{3} / m, 0\right)^{t}$. Likewise for $V_{2}: V_{2}=C\left(0,0,0,-1,-q^{3} / m, 0\right)^{t}$ and $V_{1}=S(0,0,0,1,0,0)^{t}$.

At last $V_{1}$ and $V_{2}$ satisfy: $\lambda_{1} V_{1}(q, m)+\lambda_{2} V_{2}(q, m)=0$ implies $\lambda_{1}=\lambda_{2}=0$.

Lemma 4.8 For all $j=1, \ldots, N-1$ we have

$$
\left(\prod_{i=j}^{1} H\left(q_{i}, b_{i}, m_{i}\right)\right) \cdot e_{4}=\alpha_{j}(\sqrt{\lambda}) V_{1}\left(q_{j}, m_{j}\right)+\beta_{j}(\sqrt{\lambda}) V_{2}\left(q_{j}, m_{j}\right)
$$

where $V_{k}(q, m), k=1,2$ are introduced in Definition 4.6 and $e_{4}$ in Theorem 4.5, $\alpha_{j}(),. \beta($.$) are$ trigonometrical polynomials which only depend on $q_{i}, b_{i}, m_{i}, i=1 \ldots, j$.

Moreover, there exists a constant $d_{j}>0$ (which only depends on the material constants) such that the Wronskian $W_{j}(x)=\alpha_{j}(x) \beta_{j}^{\prime}(x)-\alpha_{j}^{\prime}(x) \beta_{j}(x)$ satisfies

$$
W_{j}(x) \geq d_{j}>0, \forall x \in \mathbb{R} .
$$

Proof. We argue by iteration. We suppose that $j=1$. By Definition 4.6 and Lemma 4.7, it holds:

$$
H\left(q_{1}, b_{1}, m_{1}\right) e_{4}=\cos \left(b_{1} \sqrt{\lambda}\right) V_{1}\left(q_{1}, m_{1}\right)+\sin \left(b_{1} \sqrt{\lambda}\right) V_{2}\left(q_{1}, m_{1}\right) .
$$

Thus (59) holds for $j=1$ with $\alpha_{1}(x)=\cos \left(b_{1} x\right), \beta_{1}(x)=\sin \left(b_{1} x\right)$. Since $\forall x \in \mathbb{R}, W_{1}(x)=b_{1}$, then, for $j=1,(60)$ is true with $d_{1}=b_{1}>0$.

Now, suppose that (59) holds for $j-1$ and that there exists a constant $d_{j-1}$ such that: 
$\forall x \in \mathbb{R}, W_{j-1}(x) \geq d_{j-1}>0$.

Thus, with (58) of Lemma 4.7 we may write:

$$
\begin{aligned}
\left(\prod_{i=j}^{1} H\left(q_{i}, b_{i}, m_{i}\right)\right) e_{4} & =H\left(q_{j}, b_{j}, m_{j}\right)\left(\alpha_{j-1}(\sqrt{\lambda}) V_{1}\left(q_{j-1}, m_{j-1}\right)\right. \\
& \left.+\beta_{j-1}(\sqrt{\lambda}) V_{2}\left(q_{j-1}, m_{j-1}\right)\right) \\
& =\left(\cos \left(b_{j} \sqrt{\lambda}\right) C\left(q_{j}, m_{j}\right)+\sin \left(b_{j} \sqrt{\lambda}\right) S\left(q_{j}, m_{j}\right)\right) \\
& \times\left(\alpha_{j-1}(\sqrt{\lambda}) V_{1}\left(q_{j-1}, m_{j-1}\right)+\beta_{j-1}(\sqrt{\lambda}) V_{2}\left(q_{j-1}, m_{j-1}\right)\right) .
\end{aligned}
$$

Now, from Lemma 4.7 , we know that there exist constants $z_{i}, i=1, \ldots 8$. such that

$$
\begin{aligned}
& C\left(q_{j}, m_{j}\right) V_{1}\left(q_{j-1}, m_{j-1}\right)=z_{1} V_{1}\left(q_{j}, m_{j}\right)+z_{2} V_{2}\left(q_{j}, m_{j}\right), \\
& C\left(q_{j}, m_{j}\right) V_{2}\left(q_{j-1}, m_{j-1}\right)=z_{3} V_{1}\left(q_{j}, m_{j}\right)+z_{4} V_{2}\left(q_{j}, m_{j}\right), \\
& S\left(q_{j}, m_{j}\right) V_{1}\left(q_{j-1}, m_{j-1}\right)=z_{5} V_{1}\left(q_{j}, m_{j}\right)+z_{6} V_{2}\left(q_{j}, m_{j}\right), \\
& S\left(q_{j}, m_{j}\right) V_{1}\left(q_{j-1}, m_{j-1}\right)=z_{7} V_{1}\left(q_{j}, m_{j}\right)+z_{8} V_{2}\left(q_{j}, m_{j}\right) .
\end{aligned}
$$

Using the expressions of $C\left(q_{j}, m_{j}\right), S\left(q_{j}, m_{j}\right), V_{1}\left(q_{j-1}, m_{j-1}\right)$ and $V_{2}\left(q_{j-1}, m_{j-1}\right)$ given in Definition 4.6 we get after some computations:

$$
\begin{aligned}
& z_{1}=z_{6}=\frac{\left(m_{j-1} q_{j}+m_{j} q_{j-1}\right)\left(m_{j-1} q_{j}^{3}+m_{j} q_{j-1}^{3}\right)}{m_{j-1} q_{j}^{3}}, \\
& z_{2}=z_{3}=-z_{5}=z_{8}=\frac{m_{j} q_{j-1}\left(q_{j}^{2}-q_{j-1}^{2}\right)}{m_{j-1} q_{j}^{3}}, \\
& z_{4}=-z_{7}=\frac{m_{j} q_{j-1}\left(q_{j}+q_{j-1}\right)^{2}}{m_{j-1} q_{j}^{3}} .
\end{aligned}
$$

Using (62) in the development of the last expression of (61)

$$
\left(\prod_{i=j}^{1} H\left(q_{i}, b_{i}, m_{i}\right)\right) e_{4}=\alpha_{j}(\sqrt{\lambda}) V_{1}\left(q_{j}, m_{j}\right)+\beta_{j}(\sqrt{\lambda}) V_{2}\left(q_{j}, m_{j}\right)
$$

with

$$
\left\{\begin{aligned}
\alpha_{j}(x) & =\cos \left(b_{j} x\right)\left(z_{1} \alpha_{j-1}(x)+z_{2} \beta_{j-1}(x)\right) \\
& +\sin \left(b_{j} x\right)\left(-z_{2} \alpha_{j-1}(x)-z_{4} \beta_{j-1}(x)\right) \\
\beta_{j}(x) & =\cos \left(b_{j} x\right)\left(z_{2} \alpha_{j-1}(x)+z_{4} \beta_{j-1}(x)\right) \\
& +\sin \left(b_{j} x\right)\left(z_{1} \alpha_{j-1}(x)+z_{2} \beta_{j-1}(x)\right) .
\end{aligned}\right.
$$

That proves (59). Thanks to (64), we compute $W_{j}(x)$ and we find:

$$
\begin{aligned}
W_{j}(x) & =b_{j}\left[\left(z_{1}^{2}+z_{2}^{2}\right) \alpha_{j-1}^{2}(x)+2 z_{2}\left(z_{1}+z_{4}\right) \alpha_{j-1}(x) \beta_{j-1}(x)+\left(z_{2}^{2}+z_{4}^{2}\right) \beta_{j-1}^{2}(x)\right] \\
& +\left(z_{1} z_{4}-z_{2}^{2}\right) W_{j-1}(x) .
\end{aligned}
$$

Since $\left(z_{1}^{2}+z_{2}^{2}\right)\left(z_{2}^{2}+z_{4}^{2}\right)-\left[z_{2}\left(z_{1}+z_{4}\right)\right]^{2}=\left(z_{2}^{2}-z_{1} z_{4}\right)^{2} \geq 0$, we deduce from the previous identity that

$$
W_{j}(x) \geq\left(z_{1} z_{4}-z_{2}^{2}\right) W_{j-1}(x)
$$

From (63) we find that 


$$
\left(z_{1} z_{4}-z_{2}^{2}\right)=\frac{m_{j} q_{j-1}\left(q_{j-1}+q_{j}\right)^{2}\left(m_{j} q_{j-1}^{2}+m_{j-1} q_{j}^{2}\right)^{2}}{m_{j-1}^{3} q_{j}^{7}}>0
$$

Due to these last two inequalities, we get the conclusion :

$$
d_{j}=\left(z_{1} z_{4}-z_{2}^{2}\right) d_{j-1}>0 .
$$

Lemma 4.9 (asymptotic behaviour of the characteristic equation)

Assume that the characteristic equation is still given by Theorem 4.5. Then there exists a constant $K$ which is independent of the variable $\lambda$ such that:

where

$$
f(\sqrt{\lambda})=e^{K \sqrt{\lambda}} \cdot\left(f_{\infty}(\sqrt{\lambda})+g(\sqrt{\lambda})\right)
$$

$$
f_{\infty}(\sqrt{\lambda})=e_{4}^{t} \cdot\left(\prod_{i=N}^{1} H\left(q_{i}, b_{i}, m_{i}\right)\right) \cdot e_{4}
$$

with $e_{4}^{t}=(0,0,0,1,0,0), H\left(q_{i}, b_{i}, m_{i}\right), V_{1}(q, m)$ and $V_{2}(q, m)$ given in Definition 4.6. The function $g$ satisfies $\lim _{\lambda \rightarrow+\infty} g(\sqrt{\lambda})=0$.

Thus, the asymptotic behaviour of the spectrum $\sigma(\mathcal{A})$ corresponds to the roots of the asymptotic characteristic equation

$$
f_{\infty}(\sqrt{\lambda})=0 \text {. }
$$

These roots are all simple. Moreover, there exists a constant $d>0$ (which depends only on the material constants) such that for any root $x_{0}$ of $f_{\infty}$

$$
\left|f_{\infty}^{\prime}\left(x_{0}\right)\right| \geq d
$$

\section{Proof.}

- The first step is to prove the existence of the form $e^{K \sqrt{\lambda}} \cdot\left(f_{\infty}(\sqrt{\lambda})+g(\sqrt{\lambda})\right)$ for $f(\sqrt{\lambda})$ : each $\operatorname{ext}\left(A_{j}\right)$ is of the form $\operatorname{ext}\left(A_{j}\right)=e^{b_{j} \sqrt{\lambda}} H\left(q_{j}, b_{j}, m_{j}\right)+o\left(e^{b_{j} \sqrt{\lambda}}\right)(\mathrm{cf}$. Lemma 4.7) with $H(q, b, m)=O(1)$.

Multiplying these expressions where $j$ varies between 1 and $N$, we get: $e^{K \sqrt{\lambda}} \cdot\left(\prod_{j=N}^{1} H\left(q_{j}, b_{j}, m_{j}\right)\right)+o(K \sqrt{\lambda})$ with $K=\sum_{i=1^{N}} b_{i}$.

- The second step is to compute $f_{\infty}(\sqrt{\lambda})=e_{4}^{t} \cdot\left(\prod_{i=N}^{1} H\left(q_{i}, b_{i}, m_{i}\right)\right) \cdot e_{4}$ using Lemma 4.8 and its proof. Since $e_{4}^{t} \cdot V_{1}(q, m)=1$ and $e_{4}^{t} \cdot V_{2}(q, m)=0$

$$
f_{\infty}(\sqrt{\lambda})=e_{4}^{t} \cdot H\left(q_{N}, b_{N}, m_{N}\right) \cdot\left(\prod_{i=N-1}^{1} H\left(q_{i}, b_{i}, m_{i}\right)\right) \cdot e_{4}=f_{N} c_{N}+g_{N} s_{N}
$$

with $f_{N}=z_{1} \alpha_{N-1}+z_{2} \beta_{N-1}$ and $g_{N}=-z_{2} \alpha_{N-1}-z_{4} \beta_{N-1}$. And the wronskian $W\left(f_{N}, g_{N}\right)$ satisfies: $W\left(f_{N}, g_{N}\right)=\left(z_{2}^{2}-z_{1} z_{4}\right) W_{N-1} \leq d<0$ with $d=\left(z_{2}^{2}-z_{1} z_{4}\right) d_{N-1}$ and $d_{N-1}$ the constant introduced in Lemma 4.8 (depending only on the material constants). 
- The third step is to compute the derivative of $f_{\infty}(x)$ (for the sake of completeness, we give the proof which is exactly the same one as in [16]).

$$
f_{\infty}^{\prime}(x)=\cos \left(b_{N} x\right)\left[f_{N}^{\prime}(x)+b_{N} g_{N}(x)\right]+\sin \left(b_{N} x\right)\left[g_{N}^{\prime}(x)-b_{N} f_{N}(x)\right] .
$$

We deduce that for all $x \in \mathbb{R}, \Delta(x)=\left(f_{\infty}(x)\right)^{2}+\left(f_{\infty}^{\prime}(x)\right)^{2}$ has the following form:

$$
\Delta(x)=\left(\cos \left(b_{N} x\right) \sin \left(b_{N} x\right)\right) M(x)\left(\begin{array}{c}
\cos \left(b_{N} x\right) \\
\sin \left(b_{N} x\right)
\end{array}\right),
$$

where the matrix $M(x)$ is symmetric, positive and given by

$$
M(x)=\left(\begin{array}{ll}
M_{11}(x) & M_{12}(x) \\
M_{21}(x) & M_{22}(x)
\end{array}\right)
$$

and

$$
\left\{\begin{array}{l}
M_{11}(x)=f_{N}(x)^{2}+b_{N}^{2} g_{N}(x)^{2}+2 b_{N} g_{N}(x) f_{N}^{\prime}(x)+f_{N}^{\prime}(x)^{2}, \\
M_{12}(x)=\left(1-b_{N}^{2}\right) f_{N}(x) g_{N}(x)-b_{N}\left(f_{N}(x) f_{N}^{\prime}(x)-g_{N}(x) g_{N}^{\prime}(x)\right)+f_{N}^{\prime}(x) g_{N}^{\prime}(x), \\
M_{21}(x)=M_{12}(x) \\
M_{22}(x)=b_{N}^{2} f_{N}(x)^{2}+g_{N}(x)^{2}-2 b_{N} f_{N}(x) g_{N}^{\prime}(x)+g_{N}^{\prime}(x)^{2} .
\end{array}\right.
$$

Let $\lambda_{\min }(x), \lambda_{\max }(x)$ be the two eigenvalues of $M(x)$ such that $0 \leq \lambda_{\min }(x) \leq \lambda_{\max }(x)$. After some computation we find

$$
\begin{aligned}
\lambda_{\min }(x) \lambda_{\max }(x) & =\operatorname{det}(M(x)) \\
& =b_{N}^{2}\left(f_{N}(x)^{2}+g_{N}(x)^{2}\right)^{2}-2 b_{N}\left(f_{N}(x)^{2}+g_{N}(x)^{2}\right) W\left(f_{N}, g_{N}\right)(x) \\
& +W\left(f_{N}, g_{N}\right)(x)^{2} . \\
& =\left(W\left(f_{N}, g_{N}\right)(x)+b_{N}\left(f_{N}(x)^{2}+g_{N}(x)^{2}\right)\right)^{2} .
\end{aligned}
$$

Consequently with the estimate of the wronskian given at the end of the first step,

$$
\forall x \in \mathbb{R}, \operatorname{det}(M(x))=\lambda_{\min }(x) \lambda_{\max }(x) \geq W\left(f_{N}, g_{N}\right)(x)^{2} \geq d^{2} .
$$

On the other hand, since $f_{N}$ and $g_{N}$ are trigonometric polynomials, the trace of $M(x)$ is bounded on $\mathbb{R}$. Thus, there exists $d^{\prime}>0$ such that

$$
\forall x \in \mathbb{R}, 0 \leq \operatorname{tr}(M(x))=\lambda_{\min }(x)+\lambda_{\max }(x) \leq d^{\prime 2} .
$$

From (70) and (71) we deduce that $\lambda_{\min }(x) \geq\left(\frac{d}{d^{\prime}}\right)^{2}>0$. Therefore from (69) we get

$$
\forall x \in \mathbb{R}, \Delta(x) \geq\left(\frac{d}{d^{\prime}}\right)^{2}>0
$$

That means that if $x_{0}$ is a root of $f_{\infty}$ then $\left|f_{\infty}^{\prime}\left(x_{0}\right)\right| \geq \frac{d}{d^{\prime}}>0$. 
All the required properties are now proved to state the main result of this section:

Theorem 4.10 (the spectral gap)

Let $\lambda_{k}^{2}, k \in \mathbb{N}^{*},\left(\lambda_{k}>0\right)$ be the (strictly) monotone increasing sequence of eigenvalues of Problem $(E P)$ given at the beginning of Section 4 then

$$
\lim _{k \rightarrow+\infty}\left(\lambda_{k+1}-\lambda_{k}\right)=+\infty
$$

Proof. Since all the roots of $f_{\infty}$ are simple and since there exists a constant $d>0$ (which depends only on the material constants) such that for any root $x_{0}$ of $f_{\infty}$

$$
\left|f_{\infty}^{\prime}\left(x_{0}\right)\right| \geq d
$$

it holds $\sqrt{\lambda_{k+1}}-\sqrt{\lambda_{k}}>\sigma$ with $\sigma>0$ (cf. Theorem 5.3 of [14]).

Now $\lambda_{k+1}-\lambda_{k}=\left(\sqrt{\lambda_{k+1}}-\sqrt{\lambda_{k}}\right)\left(\sqrt{\lambda_{k+1}}+\sqrt{\lambda_{k}}\right)$ with $\lim _{k \rightarrow+\infty} \lambda_{k}=+\infty$, hence the announced result.

\section{Proof of a uniform estimate for the eigenfunctions}

Lemma 5.1 (uniform estimate for $\left|\phi_{N}\left(l_{N}\right)\right|$ )

Consider the eigenvalue problem (EP) given in Section 4. For any eigenfunction $\phi$ associated to the eigenvalue $\lambda^{2}$, there exists a constant $K_{1}$ such that:

$$
K_{1} \cdot\|\phi\|_{\mathcal{H}}^{2} \leq\left|\phi_{N}\left(l_{N}\right)\right|^{2}
$$

with the norm $\|\cdot\|_{\mathcal{H}}$ introduced in Section 2.3.1.

The proof of the lemma requires some technical intermediate results.

Let us first introduce some useful notation for the following.

\section{Notation.}

Keeping the notation $a_{j}$ and $l_{j}$ introduced in Section 2 as well as $q_{j}=\left(m_{j} / a_{j}\right)^{1 / 4}, b_{j}=q_{j} l_{j}$ introduced in Section 4.1, consider the functions $h_{i}\left(a_{j}, b_{j}, \lambda, x\right)$ for $i \in\{1 ; 2 ; 3 ; 4\}$ and $x \in\left[0 ; l_{j}\right]$ denoted $h_{i}(x)$ for the sake of simplicity:

$$
\left\{\begin{array}{l}
h_{1}(x)=\cos \left(q_{j} \sqrt{\lambda} x\right), \\
h_{2}(x)=\sin \left(q_{j} \sqrt{\lambda} x\right), \\
h_{3}(x)=\exp \left(-b_{j} \sqrt{\lambda}\right) \exp \left(q_{j} \sqrt{\lambda} x\right), \\
h_{4}(x)=\exp \left(-q_{j} \sqrt{\lambda} x\right) .
\end{array}\right.
$$

$G\left(b_{j}, q_{j}\right)$ is the $4 \times 4$ Gram matrix defined by $\left(G\left(b_{j}, q_{j}\right)\right)_{i, k}=\int_{0}^{l_{j}} h_{i}(x) h_{k}(x) d x$.

At last the matrices $D$ and $B_{1}$ are: 


$$
\begin{gathered}
D(a, q)=\frac{1}{4}\left(\begin{array}{cccc}
2 & 0 & -\frac{2}{a q^{2}} & 0 \\
0 & \frac{2}{q} & 0 & -\frac{2}{a q^{3}} \\
1 & -\frac{1}{q} & \frac{1}{a q^{2}} & -\frac{1}{a q^{3}} \\
e^{b \sqrt{\lambda}} & \frac{e^{b \sqrt{\lambda}}}{q} & \frac{e^{b \sqrt{\lambda}}}{a q^{2}} & \frac{e^{b \sqrt{\lambda}}}{a q^{3}}
\end{array}\right) \\
B_{1}(q, m)=\frac{1}{4}\left(\begin{array}{cccc}
1 & \frac{1}{q} & \frac{q^{2}}{m} & \frac{q}{m} \\
\frac{q}{m} & 1 & \frac{q^{3}}{m} & \frac{q^{2}}{m} \\
\frac{m}{q^{2}} & \frac{m}{q^{3}} & 1 & \frac{1}{q} \\
\frac{m}{q} & \frac{m}{q^{2}} & q & 1
\end{array}\right) .
\end{gathered}
$$

\section{Lemma 5.2}

Any eigenfunction $\phi$ associated to the eigenvalue $\lambda^{2}$ for the eigenvalue problem $(E P)$ given at the beginning of Section 4 may be uniquely written as a linear combination of the $\left(h_{i}\right)$ 's. Denote by $\left(C_{j}\right)_{i}$ the coefficients of the decomposition of $\phi_{j}$ in the basis $\left(h_{i}\right)_{i \in\{1 ; 2 ; 3 ; 4\}}$

i.e. $\phi_{j}(x)=\sum_{i=1}^{4}\left(C_{j}\right)_{i} h_{i}(x)$ for $j \in\{1, \cdots, N\}$ and $x \in\left[0, l_{j}\right]$. Then

$$
C_{j}=D\left(a_{j}, q_{j}\right) V_{j}(0), \text { and } A(q, b, m)=\frac{1}{4} \exp (b \sqrt{\lambda}) B_{1}(q, m)
$$

with $V_{j}$ and $A(q, b, m)$ defined in Section 4.1.

( $V_{j}$ being computed for the $j-$ th component of the particular eigenfunction $\left.\phi\right)$.

There exists a positive constant $C$ (by constant we mean independent of $\lambda$ ) such that

$$
\|\phi\|_{\mathcal{H}}^{2}=\sum_{j=1}^{N} \int_{0}^{l_{j}}\left|\phi_{j}(x)\right|^{2} d x \leq C \max _{j \in\{1 \cdots N\}}\left(C_{j}^{t} C_{j}\right) .
$$

Proof. Proving that the $h_{i}$ 's are linearly independent is a classical computation. (78) is proved by calculation.

By definition of the inner product in $\mathcal{H}$ (section 2.3.1):

$$
\|\phi\|_{\mathcal{H}}^{2}=\sum_{j=1}^{N} \int_{0}^{l_{j}} \phi_{j}(x)^{2} d x=\sum_{j=1}^{N} C_{j}^{t} G\left(b_{j}, q_{j}\right) C_{j} .
$$

Now, after calculation, the matrix $G(b, q)$ is:

$$
\begin{gathered}
G(b, q)=\frac{1}{2 q \sqrt{\lambda}} \times \\
\left(\begin{array}{cccc}
c+b \sqrt{\lambda} & s^{2} & 1-(c-s) e^{-b \sqrt{\lambda}} & c+s-e^{-b \sqrt{\lambda}} \\
s^{2} & -c s+b \sqrt{\lambda} & 1-(c+s) e^{-b \sqrt{\lambda}} & -c+s+e^{-b \sqrt{\lambda}} \\
1-(c-s) e^{-b \sqrt{\lambda}} & 1-(c+s) e^{-b \sqrt{\lambda}} & 1-e^{-2 b \sqrt{\lambda}} & 2 b e^{-b \sqrt{\lambda}} \sqrt{\lambda} \\
c+s-e^{-b \sqrt{\lambda}} & -c+s+e^{-b \sqrt{\lambda}} & 2 b e^{-b \sqrt{\lambda}} \sqrt{\lambda} & 1-e^{-2 b \sqrt{\lambda}}
\end{array}\right)
\end{gathered}
$$


with the notation $c=\cos (b \sqrt{\lambda}), s=\sin (b \sqrt{\lambda})$.

Note that all its terms are bounded with respect to $\lambda$. The estimate of $\|\phi\|_{\mathcal{H}}^{2}$ follows.

Lemma 5.3 (estimate of $\phi_{N}\left(l_{N}\right)$ )

Let $M(\lambda)$ the $4 \times 4$ matrix defined by (48) in Section 4.1. Denote by $\alpha(\lambda)$ (respectively $\beta(\lambda)$ ) the first (resp. second) term of the first line of $M(\lambda)$ i.e.

$$
\left\{\begin{array}{l}
\alpha(\lambda)=e_{1}^{t} M(\lambda) e_{1}, \\
\beta(\lambda)=e_{1}^{t} M(\lambda) e_{2},
\end{array}\right.
$$

with $e_{1}=(1,0,0,0)$ and $e_{2}=(0,1,0,0)$.

Then the eigenfunction $\phi$ of Problem (EP) associated to the eigenvalue $\lambda^{2}$ can be chosen such that $\phi_{N}\left(l_{N}\right)=\beta(\lambda)$ and the asymptotic behaviour of $\alpha(\lambda)$ and $\beta(\lambda)$ is given by:

$$
\left\{\begin{array}{l}
\alpha(\lambda)=C \cdot q_{N} \cdot e^{B \sqrt{\lambda}}+o\left(e^{B \sqrt{\lambda}}\right), \\
\beta(\lambda)=C \cdot e^{B \sqrt{\lambda}}+o\left(e^{B \sqrt{\lambda}}\right),
\end{array}\right.
$$

with $B:=\sum_{j=1}^{N} b_{j}$.

Note that the constants $C$ are not identical nor equal to those of Lemma 5.2 but we will always call the constants $C$. All of them are independent of $\lambda$ but depend on the material constants given by the $a_{j}$ 's, $b_{j}$ 's...

Proof. Any eigenfunction $\phi$ associated to the eigenvalue $\lambda^{2}$ satisfies condition (19) of Section 2.3.1. In particular $\phi_{1}(0)=\phi_{1}^{\prime}(0)=0$ so the first two components of the vector $V_{1}(0)$ associated to $\phi$ (defined in Section 4.1) vanish. Moreover (25) and (26) also imply $\phi_{N}^{\prime \prime}\left(l_{N}\right)=0$ and $\phi_{N}^{(3)}\left(l_{N}\right)=0$ so the third and fourth components of $V_{N}\left(l_{N}\right)$ vanish : $V_{N}\left(l_{N}\right)$ is of the form $\left(\left(V_{N}\left(l_{N}\right)\right)_{1},\left(V_{N}\left(l_{N}\right)\right)_{2}, 0,0\right)^{t}$.

Now, due to Lemma 4.1, $V_{N}\left(l_{N}\right)=M(\lambda) V_{1}(0)$ or $V_{1}(0)=M(\lambda)^{-1} V_{N}\left(l_{N}\right)$. Thus, if $\mu(\lambda)$ (respectively $\nu(\lambda)$ ) is the third (resp. fourth) term of the first line of $M(\lambda)^{-1}$, then $\mu(\lambda)\left(V_{N}\left(l_{N}\right)\right)_{1}+$ $\nu(\lambda)\left(V_{N}\left(l_{N}\right)\right)_{2}=0$.

$\left(V_{N}\left(l_{N}\right)\right)_{1}=\nu(\lambda)$ and $\left(V_{N}\left(l_{N}\right)\right)_{2}=-\mu(\lambda)$ is a solution of this equation which means that the eigenfunction $\phi$ of Problem (EP) associated to the eigenvalue $\lambda^{2}$ can be chosen such that $\phi_{N}\left(l_{N}\right)=\nu(\lambda)$ (such an eigenfunction is not normalized).

Now, to avoid the use of the inverse of the matrix $M(\lambda)$, we choose to switch the indices, which is equivalent to switch the boundary conditions i.e. in that proof as well as in the proofs of Lemmas 5.4 and 5.6, the definitions of $V$ and $D(A)$ change : conditions (19), (25) and (26) of Section 2.3.1 become $\phi_{N}\left(l_{N}\right)=\phi_{N}^{\prime}\left(l_{N}\right)=0$ and $\phi_{1}^{\prime \prime}(0)=\phi_{1}^{(3)}(0)=0$. Thus the eigenfunction $\phi$ of Problem $(E P)$ associated to the eigenvalue $\lambda^{2}$ can be chosen such that $\phi_{N}\left(l_{N}\right)=\beta(\lambda)$.

The second part of the proof contains the estimate of some terms of the matrix $M(\lambda)$. Recall that 


$$
M(\lambda)=A_{N} A_{N-1} \ldots A_{2} A_{1} .
$$

The asymptotic behaviour of $A_{j}=A\left(q_{j}, b_{j}, m_{j}\right)$ is given by $A_{j}=\exp \left(b_{j} \sqrt{\lambda}\right) B_{1}\left(q_{j}, m_{j}\right)+$ $o\left(\exp \left(b_{j} \sqrt{\lambda}\right)\right)$ with $B_{1}\left(q_{j}, m_{j}\right)$ defined by the decomposition $A(q, b, m)=\sum_{\epsilon \in\{-1 ; 0 ; 1\}} \exp (\epsilon b \sqrt{\lambda}) B_{\epsilon}(q, m)$.

Combining the above estimates and using (48) lead to the desired result.

The aim is still the estimation of $\|\phi\|_{\mathcal{H}}$ which requires, due to Lemma 5.2, the estimation of $C_{j}$.

Lemma 5.4 (estimate of $C_{j}$ )

Let $C_{j}$ be the vector already defined by (78) with a fixed $j \in\{1, \cdots, N\}$, the vector $\vec{b}$ be defined by $\vec{b}=\left(b_{1}, \cdots, b_{N}\right)$ and denote by $\vec{u} \cdot \vec{v}:=\sum_{i=1}^{N} u_{i} v_{i}$, then there exist vectors $W_{\vec{\epsilon}}(\lambda)$ such that $C_{j}$ is of the form

$$
C_{j}:=\sum_{\vec{\epsilon} \in\{-2 ;-1 ; 0 ; 1\}^{N}} e^{\vec{b} \cdot \vec{\epsilon} \sqrt{\lambda}} W_{\vec{\epsilon}}(\lambda)
$$

and all the terms of $W_{\vec{\epsilon}}(\lambda)$ are dominated by the function $\lambda \mapsto e^{B \sqrt{\lambda}}$ asymptotically (with $\left.B:=\sum_{j=1}^{N} b_{j}\right)$.

More precisely the terms of $W_{\vec{\epsilon}}(\lambda)$ only contain expressions of the form $\cos \left(b_{j} \sqrt{\lambda}\right)$ and $\sin \left(b_{j} \sqrt{\lambda}\right)$ with $j \in\{1, \ldots, N\}$.

\section{Proof.}

First Part. For a fixed $j \in\{1, \cdots, N\}$, we start with isolating the terms containing $e^{b_{j} \sqrt{\lambda}}$ in the involved matrices.

$$
\left\{\begin{array}{l}
D\left(a_{j}, q_{j}\right)=e^{b_{j} \sqrt{\lambda}} D^{+}\left(a_{j}, q_{j}\right)+D^{r}\left(a_{j}, q_{j}\right) \\
A_{j}=A\left(q_{j}, b_{j}, m_{j}\right)=e^{b_{j} \sqrt{\lambda}} B^{+}\left(q_{j}, m_{j}\right)+B^{r}\left(q_{j}, m_{j}\right) .
\end{array}\right.
$$

The decomposition of $A_{j}$ is the same one as in the proof of Lemma 5.3 i.e. the matrix called $B^{+}$in that proof is $B_{1}$. The exponent $r$ is chosen for the rest which does not contain $e^{b_{j} \sqrt{\lambda}}$. Since $M(\lambda)=A_{N} \cdots A_{j} \cdots A_{2} A_{1}$ (cf. (48)), it holds:

$$
\begin{aligned}
M(\lambda) & =A_{N} \cdots A_{j+1}\left(e^{b_{j} \sqrt{\lambda}} B^{+}\left(q_{j}, m_{j}\right)+B^{r}\left(q_{j}, m_{j}\right)\right) A_{j-1} \cdots A_{2} A_{1} \\
& =e^{b_{j} \sqrt{\lambda}} A_{N} \cdots A_{j+1} B^{+}\left(q_{j}, m_{j}\right) A_{j-1} \cdots A_{2} A_{1}+A_{N} \cdots A_{j+1} B^{r}\left(q_{j}, m_{j}\right) A_{j-1} \cdots A_{2} A_{1} \\
& =: e^{b_{j} \sqrt{\lambda}} M^{+}(\lambda)+M^{r}(\lambda) .
\end{aligned}
$$

The first and second terms of the first line of the matrix $M(\lambda)$ denoted by $\alpha$ and $\beta$ in Lemma 5.3 can be decomposed as follows: 


$$
\left\{\begin{array}{l}
\alpha(\lambda)=e_{1}^{t} M(\lambda) e_{1}=e^{b_{j} \sqrt{\lambda}} e_{1}^{t} M^{+}(\lambda) e_{1}+e_{1}^{t} M^{r}(\lambda) e_{1}=e^{b_{j} \sqrt{\lambda}} \alpha^{+}(\lambda)+\alpha^{r}(\lambda), \\
\beta(\lambda)=e_{1}^{t} M(\lambda) e_{2}=e^{b_{j} \sqrt{\lambda}} \beta^{+}(\lambda)+\beta^{r}(\lambda) .
\end{array}\right.
$$

Thus the vector $V_{1}(0)$ is decomposed as well: $V_{1}(0)=(\beta(\lambda),-\alpha(\lambda), 0,0)^{t}=e^{b_{1} \sqrt{\lambda}} V_{1}^{+}(0)+V_{1}^{r}(0)$ with $V_{1}^{+}(0)=\left(\beta^{+}(\lambda),-\alpha^{+}(\lambda), 0,0\right)^{t}$. Then $C_{j}=D\left(a_{j}, q_{j}\right) A_{j-1} \cdots A_{1} V_{1}(0)$ may be written as:

$$
C_{j}=e^{2 b_{j} \sqrt{\lambda}} C_{j}^{++}+e^{b_{j} \sqrt{\lambda}} C_{j}^{+}+C_{j}^{r} \text { with } C_{j}^{++}:=D^{+}\left(a_{j}\right) A_{j-1} \cdots A_{1} V_{1}^{+}(0)
$$

where neither $C_{j}^{++}$, nor $C_{j}^{+}$, nor $C_{j}^{r}$ contains $e^{b_{j} \sqrt{\lambda}}$. The vanishing of $C_{j}^{++}$remains to be proved in order to establish (80).

Second Part. For a fixed $j \in\{1, \cdots, N\}$, let us prove that $C_{j}^{++}=0$ with $C_{j}^{++}$defined by (81). Recall that $M(\lambda)=e^{b_{j} \sqrt{\lambda}} M^{+}(\lambda)+M^{r}(\lambda)$ with $M^{+}(\lambda)=A_{N} \cdots A_{j+1} B^{+}\left(q_{j}, m_{j}\right) A_{j-1} \cdots A_{2} A_{1}$. The matrices $A_{i}$ for $i \in\{1, \cdots, j-1\}$ defined in Section 4.1 are all invertible since their determinant is equal to 1 (calculation).

The matrix $B^{+}\left(q_{j}, m_{j}\right)$ is defined as follows:

$$
B^{+}(q, m)=\frac{1}{4}\left(\begin{array}{cccc}
1 & \frac{1}{q} & \frac{q^{2}}{m} & \frac{q}{m} \\
q & 1 & \frac{q^{3}}{m} & \frac{q^{2}}{m} \\
\frac{m}{q^{2}} & \frac{m}{q^{3}} & 1 & \frac{1}{q} \\
\frac{m}{q} & \frac{m}{q^{2}} & q & 1
\end{array}\right) .
$$

Note that the columns of $B^{+}\left(q_{j}, m_{j}\right)$ are all proportional to the first one so the rank of $B^{+}\left(q_{j}, m_{j}\right)$ is 1 . Thus the rank of $M^{+}(\lambda)$ is also 1 which means in particular that all its lines are proportional to the first one.

Now the first (respectively second) term of the first line of $M^{+}(\lambda)$ is, by definition, $\alpha^{+}(\lambda)$ (resp. $\left.\beta^{+}(\lambda)\right)$ and $V_{1}^{+}(0)=\left(\beta^{+}(\lambda),-\alpha^{+}(\lambda), 0,0\right)^{t}$. So the first term of the product $M^{+}(\lambda) V_{1}^{+}(0)$ is 0 . And since the other lines of $M^{+}(\lambda)$ are proportional to the first one, the other terms also vanish i.e. $M^{+}(\lambda) V_{1}^{+}(0)=0$.

It is equivalent to $A_{N} \cdots A_{j+1} B^{+}\left(q_{j}, m_{j}\right) A_{j-1} \cdots A_{2} A_{1} V_{1}^{+}(0)=0$ and, since $\left(A_{N} \cdots A_{j+1}\right)$ is invertible, it implies: $A_{j-1} \cdots A_{2} A_{1} V_{1}^{+}(0) \in \operatorname{Ker}\left(B^{+}\left(q_{j}, m_{j}\right)\right)$.

The matrix $D^{+}\left(a_{j}, q_{j}\right)$ is defined as follows:

$$
D^{+}\left(a_{j}, q_{j}\right)=\frac{1}{4}\left(\begin{array}{cccc}
0 & 0 & 0 & 0 \\
0 & 0 & 0 & 0 \\
0 & 0 & 0 & 0 \\
1 & \frac{1}{q_{j}} & \frac{1}{a_{j} q_{j}^{2}} & \frac{1}{a_{j} q_{j}^{3}}
\end{array}\right) .
$$

Since $q_{j}^{2} / m_{j}=1 /\left(a_{j} q_{j}^{2}\right)$, it clearly holds $\operatorname{Ker}\left(B^{+}\left(q_{j}, m_{j}\right)\right)=\operatorname{Ker}\left(D^{+}\left(a_{j}, q_{j}\right)\right)$. Thus $C_{j}^{++}:=D^{+}\left(a_{j}, q_{j}\right) A_{j-1} \cdots A_{2} A_{1} V_{1}^{+}(0)=0$. 
Lemma 5.5 (estimate of $V_{j}\left(l_{j}\right)$ )

Let $j \in\{1, \cdots, N\}$ and let the vector $V_{j}$ be defined as in Section 4.1. For any $K>0$, there exists a positive constant $C$ (independent of $\lambda$ ) such that, if $\lambda>K$, for $j \in\{1, \cdots, N\}$ and $i \in\{1 ; 2 ; 3 ; 4\}$

$$
\left|e_{i}^{t} V_{j}\left(l_{j}\right)\right| \leq C e^{B \sqrt{\lambda}}
$$

with $e_{1}^{t}=(1,0,0,0), e_{2}^{t}=(0,1,0,0), e_{3}^{t}=(0,0,1,0), e_{4}^{t}=(0,0,0,1)$ and $B:=\sum_{j=1}^{N} b_{j}$.

The constants are still all denoted by $C$. They only depend on the material constants.

Proof. The property is proved by induction. The vector $V_{1}(0)$ is: $V_{1}(0)=(\beta(\lambda),-\alpha(\lambda), 0,0)^{t}$ and the behaviour of $\alpha$ and $\beta$ is given by Lemma 5.3. The components of $V_{1}\left(l_{1}\right)=A_{1} V_{1}(0)$ keep the same fastest growing term as the terms of $V_{1}(0)$. Indeed the multiplication by the matrix $A_{1}$ which contains exponential terms could a priori change the exponential into $e^{\left(2 b_{1}+\sum_{j=2}^{N} b_{j}\right) \sqrt{\lambda}}$ but as it was proved for $C_{j}$ in the proof of Lemma 5.4, it is not the case.

Lemma 5.6 (a more precise estimate of $C_{j}$ )

Let $C_{j}$ be the vector already defined by (78) with a fixed $j \in\{1, \cdots, N\}$. For any $K>0$, there exists a constant $C$ (independent of $\lambda$ ) such that, if $\lambda>K$ and $i \in\{1 ; 2 ; 3 ; 4\}$ :

$$
\left|\left(C_{j}\right)_{i}\right| \leq C e^{B \sqrt{\lambda}}
$$

with $\left(C_{j}\right)_{i}$ the $i-$ th term of the vector $C_{j}$ as in Lemma 5.2 and $B:=\sum_{j=1}^{N} b_{j}$.

Proof. Recall that $C_{j}=D\left(a_{j}, q_{j}\right) V_{j}(0)$ (cf. (78)). We have just proved in the second part of the proof of Lemma 5.5 that, for any $j \in\{1, \cdots, N\}$, the absolute values of the four terms of $V_{j+1}(0)$ are bounded by $C e^{B \sqrt{\lambda}}$ for large values of $\lambda$. It is also clear for $V_{1}(0)=(\beta(\lambda),-\alpha(\lambda), 0,0)^{t}$ due to the estimates of $\alpha$ and $\beta$ given in Lemma 5.3.

Now the matrix $D\left(a_{j}, q_{j}\right)$ contains exponential terms but we proved in the proof of Lemma 5.4 that they do not affect the fastest growing term of $C_{j}$. Hence the result.

Theorem 5.7 (first uniform estimate for $\left|\phi_{N}\left(l_{N}\right)\right|$ )

Consider the eigenvalue problem (EP) given in Section 3.1. For any eigenfunction $\phi \in D(A)$ associated to the eigenvalue $\lambda^{2}$, there exist a constant $K_{1}$ such that:

$$
K_{1} \cdot\|\phi\|_{\mathcal{H}}^{2} \leq\left|\phi_{N}\left(l_{N}\right)\right|^{2}
$$

with the norm $\|\cdot\|_{\mathcal{H}}$ introduced in Section 2.3.1.

Proof. Due to Lemma 5.2

$$
\|\phi\|_{\mathcal{H}}^{2} \leq C \max _{j \in\{1 \cdots N\}}\left(C_{j}^{t} C_{j}\right) .
$$

Then (84) implies $\|\phi\|_{\mathcal{H}}^{2} \leq C\left(e^{B \sqrt{\lambda}}\right)^{2}$.

Now, we stated in the proof of Theorem 5.3 that the eigenfunction $\phi$ of Problem $(E P)$ associated to the eigenvalue $\lambda^{2}$ can be chosen such that $\phi_{N}\left(l_{N}\right)=\beta(\lambda)$. The estimate of $\beta(\lambda)$ for large values of $\lambda$ given by Theorem 5.3 gives the desired result. 


\subsection{Second estimate: admissibility}

Theorem 5.8 (second estimate for controllability)

Consider the eigenvalue problem (EP) associated to Problem $(P)$ (given in Section 4). For any eigenfunction $\phi$ associated to the eigenvalue $\lambda^{2}$, there exists a constant $K_{2}$ such that:

$$
\left|\phi_{N}\left(l_{N}\right)\right|^{2} \leq K_{2} \cdot\|\phi\|_{\mathcal{H}}^{2}
$$

with the norm $\|\cdot\|_{\mathcal{H}}$ defined in Section (2.3.1).

Proof. We established in the proof of Lemma 5.2

$$
\|\phi\|_{\mathcal{H}}^{2}=\sum_{j=1}^{N} \int_{0}^{l_{j}} \phi_{j}(x)^{2} d x=\sum_{j=1}^{N} C_{j}^{t} G\left(b_{j}, q_{j}\right) C_{j}
$$

with $C_{j}$ and $G\left(b_{j}, q_{j}\right)$ defined in the same Lemma. Thus $\|\phi\|_{\mathcal{H}}^{2} \geq C_{1}^{t} G\left(b_{1}, q_{1}\right) C_{1}$ and it remains to estimate this expression from below.

Due to $(78)$ it holds $C_{1}=D\left(a_{1}, q_{1}\right) V_{1}(0)$ and we stated in Lemma 5.3 that

$$
V_{1}(0)=(\beta(\lambda),-\alpha(\lambda), 0,0)^{t}=\left(\beta, q_{N} \cdot \beta+o(\beta), 0,0\right)^{t}
$$

as $\lambda$ and thus $\beta$ tend to infinity. Then, multiplying by the matrix $D\left(a_{1}, q_{1}\right)$ given just before Lemma 5.2, it follows

$$
C_{1}=2\left(\beta, \beta+\frac{o(\beta)}{q_{1}},-\frac{o(\beta)}{2 q_{1}}, \beta e^{b_{1} \sqrt{\lambda}}+\frac{e^{b_{1} \sqrt{\lambda}}}{2 q_{1}} \cdot o(\beta)\right) .
$$

Now we proved in Lemma 5.6 that, for any $K>0$, there exists a constant $C$ (independent of $\lambda)$ such that, if $\lambda>K$ and $i \in\{1 ; 2 ; 3 ; 4\}$, then $\left|\left(C_{j}\right)_{i}\right| \leq C \beta(\lambda)$ with $\left(C_{j}\right)_{i}$ the $i-t h$ term of the vector $C_{j}$. So the fourth term of $C_{1}$ grows as fast as $\beta$ i.e.

$$
C_{1}=2\left(\beta, \beta+\frac{o(\beta)}{q_{1}},-\frac{o(\beta)}{2 q_{1}}, O(\beta)\right) .
$$

Looking thoroughly at the terms of the matrix $G\left(b_{1}, q_{1}\right)$ given in the proof of Lemma 5.2 , we can see that only two terms do not tend to zero as $\lambda$ tends to infinity which can be written as:

$$
G\left(b_{1}, q_{1}\right)=\left(\begin{array}{cccc}
\frac{b_{1}}{2 q_{1}} & 0 & 0 & 0 \\
0 & \frac{b_{1}}{2 q_{1}} & 0 & 0 \\
0 & 0 & 0 & 0 \\
0 & 0 & 0 & 0
\end{array}\right)+o(1) .
$$

It follows $C_{1}^{t} \cdot G\left(b_{1}, q_{1}\right) \cdot C_{1}=\frac{4 b_{1}}{q_{1}} \cdot \beta^{2}+o\left(\beta^{2}\right)$ and since $\left|\phi_{N}\left(l_{N}\right)\right|^{2}=|\beta(\lambda)|^{2}$, the desired estimate follows. 


\section{Acknowledgement.}

The authors thank the referees for their attentive reading of the manuscript and the questions they asked.

\section{References}

[1] F. Ali Mehmeti. Nonlinear Waves in Networks. Akademie Verlag, Berlin, 1994.

[2] F. Ali Mehmeti. Transient Waves in Semi-Infinite Structures : the Tunnel Effect and the Sommerfeld Problem. Akademie Verlag, Berlin, 1996.

[3] F. Ali Mehmeti, J. von Below and S. Nicaise, edit. Partial differential equations on multistructures, Lecture Notes in Pure and Applied Mathematics, Marcel Dekker, 2001.

[4] K. Ammari, M. Tucsnak. Stabilization of second order evolution equations by a class of unbounded feedbacks. ESAIM Control Optim. Calc. Var. 6, p. 361-386, 2001.

[5] K. Ammari, M. Mehrenberger. Stabilization of coupled systems. Acta Math. Hungar. 123 (1-2), p. 1-10, 2009.

[6] K. Ammari, D. Mercier, V. Régnier, J. Valein. Spectral analysis and stabilization of a chain of serially connected Euler-Bernoulli beams and strings. Comm. In Pure and Appl. Anal. 11/2, p. 785-807, 2012.

[7] W. Arendt and C. J. K. Batty. Tauberian theorems and stability of one-parameter semigroups. Trans. Amer. Math. Soc., 305, p. 837-852, 1988.

[8] J. von Below. A characteristic equation associated to an eigenvalue problem on $C^{2}$ networks. Linear Algebra Appl., 71, p. 309-325, 1985.

[9] C. Castro, E. Zuazua. Analyse spectrale et contrôle d'un système hybride composé de deux poutres connectées par une masse ponctuelle. C. R. Acad. Sci. Paris, 322, p. 351-356, 1996.

[10] G. Chen, M. Delfour, A. Krall, G. Payre. Modeling, stabilization and control of serially connected beams. SIAM J. Control Opt., 25, p. 526-546, 1987.

[11] B. Dekoninck, S. Nicaise. The eigenvalue problem for network of beams. Linear Algebra and its Applications, 314, p. 165-189, 2000.

[12] A. Haraux. Séries lacunaires et contrôle semi-interne des vibrations d'une plaque rectangulaire. J. Maths Pures et Appl., 68, p. 457-465, 1989.

[13] J.E. Lagnese, G. Leugering, E.J.P.G. Schmidt. Modeling, Analysis and Control of Dynamic Elastic Multi-Link Structures. Birkhäuser, Boston, 1994.

[14] D. Mercier. Spectrum analysis of a serially connected Euler-Bernoulli beams problem. Netw. Hetereg Media, 4, p. 874-894, 2009. 
[15] D. Mercier, V. Régnier. Spectrum of a network of Euler-Bernoulli beams. J. Math. Anal. and Appl., 337/1, p.174-196, 2007.

[16] D. Mercier, V. Régnier. Boundary controllability of a chain of serially connected EulerBernoulli beams with interior masses. Collect. Math., 60/3, p. 307-334, 2009.

[17] S. Nicaise. Diffusion sur les espaces ramifiés. Thèse de doctorat, Mons, Belgique, 1986.

[18] W. H. Paulsen. The exterior matrix method for sequentially coupled fourth-order equations. J. of Sound And Vibration 308, 4, p. 132-163, 2007. 